\title{
Numerical investigation on the Hydrodynamic Performance of Fast SWATHs with Optimum Canted Struts Arrangements
}

\author{
Giuliano Vernengo ${ }^{1} \&$ Stefano Brizzolara ${ }^{2}$ \\ 1) Naval Architecture and Marine Engineering Unit, DITEN, University of Genoa, Italy \\ 2) Department of Aerospace and Ocean Engineering, Virginia Tech, Blacksburg, VA, USA
}

\begin{abstract}
Small Waterplane Area Twin Hulls (SWATHs) are known to have superior seakeeping performance but higher resistance compared to equivalent catamarans or mono-hulls. A way to improve their resistance characteristics is to use unconventional hull forms parametrically defined and optimized by CFD methods. This study builds on previous SWATH optimization studies proposing a comprehensive, systematic investigation on the effect of different shapes and canting angles of the struts. For the first time we demonstrate the importance of considering the shape of the strut that is fully parametrized in our study. The effect of the design speed on the best shape is addressed through a multi-objective optimization targeting the minimum total resistance at two very different speeds, namely the cruise and slow transfer speeds. Optimum hull shapes are discussed in terms of maximum resistance reduction, together with the predicted free waves patterns.
\end{abstract}

KEY WORDS: Unconventional Small Waterplane Area Twin Hull (SWATH); Canted multi-struts; Full parametric shape model; Multi-objective Optimization; Boundary Element Method (BEM); Wave resistance; Genetic algorithms.

\section{INTRODUCTION}

SWATH ships have proven to be excellent platforms for particular marine vehicles where low motions at sea are of primary importance: this is the case of passenger ships (Navatek, 2015), research vessels (Lang, 1986) and (NOAA, 2015) and special navy vessels (Scragg et al., 1997) among others. Recently Brizzolara et al. (2011) have introduced an innovative design of a family of autonomous surface vessels based on unconventional SWATH hull that demonstrated their superior seakeeping performance with respect to equivalent catamarans (Brizzolara \& Chryssostomidis, 2014). As a downside to the excellent seakeeping behaviors, generally conventional SWATH vessels, having torpedo-like underwater hulls based on symmetric airfoil shapes do not meet high performance in terms of calm water resistance. A way to eliminate this drawback was first introduced by Brizzolara (2004) and it relied on automatic parametric optimization algorithms exploring different unconventional shapes analytically defined that are able to maximize the positive interference effects between the wave trains generated at the bow and stern of the underwater hulls. The design by optimization method was then implemented with a fully 3D B-Spline modeling of the underwater hull (Brizzolara and Vernengo, 2011) still allowing for unconventional 'wasp-body' like shapes of the underwater 
hulls but keeping the struts unchanged.

However, the wave resistance of a SWATH does not only depend on the submerged hulls; it is in fact affected by the struts shape and configuration, i.e. the structural elements that connect the SWATH superstructure to the lower hulls. No study has been made so far about the influence of strut shape on the hydrodynamic performance of SWATH. This kind of study has to consider some hydrostatic constraints, since the struts, in addition to their structural functionality, are elements that ensure a sufficient transversal and longitudinal stability to the vessel and do contribute to the total drag by a significant fraction. An additional contribution to wave cancellation effect, though, can come out from the customized design of the struts. The number of the struts used for each side of the hull, their longitudinal position, their dihedral angle and the shape of each of them represent additional degrees of freedom to play with in order to reach the goal of optimum performance.

In this framework, we present a study on the interference effects generated by canted struts in SWATH vessels designed to achieve best resistance performance at medium to high Froude numbers. The effect of the struts on optimum drag is isolated through separate optimizations. The effect of the design speed is also investigated in the same way.

\section{CANTED MULTI-STRUTS SWATH PARAMETRIC DESIGN}

The key for a successful hydrodynamic hull form optimization relies on an efficient parametric geometry definition. A full parametric model of the proposed unconventional SWATH has been developed extending the degrees of freedom of the geometric description initially proposed in Brizzolara et al. (2011).

The main dimensions and characteristics of the unconventional SWATH design taken as reference for this study are listed in Table 1.

Table 1: Technical data of the unconventional SWATH

\begin{tabular}{lll}
\hline $\mathrm{L}_{\mathrm{OA}}$ & 7.02 & $\mathrm{~m}$ \\
$\mathrm{~L}_{\mathrm{WL}}$ & 5.90 & $\mathrm{M}$ \\
$\mathrm{B}_{\mathrm{Max}}$ & 5.44 & $\mathrm{M}$ \\
$\mathrm{T}$ & 1.18 & $\mathrm{M}$ \\
$\mathrm{D}$ & 3.33 & $\mathrm{M}$ \\
$\nabla$ & 4.34 & $\mathrm{~T}$ \\
$\mathrm{~V}_{\mathrm{Max}}$ & 12 & Knots \\
\hline
\end{tabular}

The fully parametric model of the SWATH is created using curves and surface primitives of the CAESES 3D parametric CAD (see for instance Harries, 1998). The parametric model is structured in two main blocks: one for the submerged body and the other for the two struts. The underwater part of the hull is an unconventional prolate ellipsoid whose generator curves (for the max and min semi-axes) are two B-Spline curves built on seven control points, shown in Figure 1. The extremes ones (points $P_{0}$ and $P_{6}$ ) are used to define the length overall, $L_{O A}$, of the submerged hull. The two points directly adjacent to the two extremes, $P_{1}$ and $P_{5}$, are necessary to ensure the tangency of the curve (hence of the surface); as a consequence of this geometric construction these two points are allowed to move only in the vertical direction. The three remaining inner points, $P_{2}, P_{3}$ and $P_{4}$, directly drive the principal shape modifications of the underwater hulls. These three points can be freely moved along both vertical and longitudinal directions, creating humps and hollows along the length of the hull itself. This is the main feature of the parametric model for the submerged hulls that enables reaching low resistance values: there is a particular position relative minimum area section over the hull length that ensures the most favorable wave interference effect; this phenomenon helps reducing the wave resistance of a SWATH as first demonstrated in Brizzolara (2004). In addition to these eight parameters (three longitudinal coordinates and five vertical ones), the parametric definition of the BSpline curves requires a ninth parameter. This parameter, called ellipticity factor, $B / H$, never considered before (see for example Brizzolara \& Vernengo, 2011), sets the beam to height ratio of all the transversal sections of the underwater hulls. 


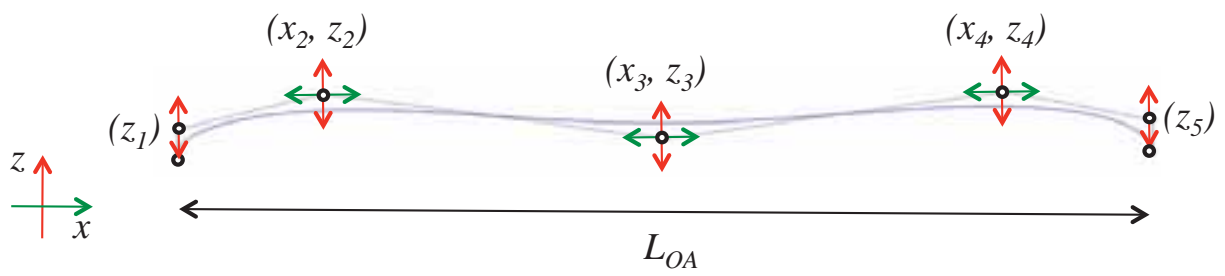

Figure 1: Parametric definition of the B-Spline curve used to generate the shape of the underwater hull of the SWATH. Allowed horizontal and vertical motions of the points are highlighted green and red arrows respectively.

The two struts are modeled like three-dimensional airfoils, as shown in Figure 2. The same parametric airfoil section (in the xy-plane) is repeated over the height of the struts. The section, of course, can differ for the aft and the forward strut, and it is generated on the basis of classic airfoil theory: the value and the position of the maximum thickness, $X_{M}$ and $Z_{M}$ respectively, the entrance and the exit angles of the section, $\alpha_{\text {Entrance }}$ and $\alpha_{E x i t}$ respectively, the positions of the foremost extreme, $X_{F o r w}$, and the section chord, $c$, are used as free parameters of the section. In addition to these parameters there are others controlling the global shape of the two struts: the transversal position of their leading edges with respect to the centerline of the submerged hull, $Y_{\text {Transl }}$, and the canting angles with respect to the vertical direction, $\vartheta$, as shown in Figure 3; the rotation of the symmetry plane of the struts is centered around the longitudinal axis of the underwater hull, in such a way that vertical struts correspond to $\vartheta=0$. The transverse sections of the struts are actually translated in their horizontal plane ( $x y$ plane). The aft and forward struts are inclined by the same angle.

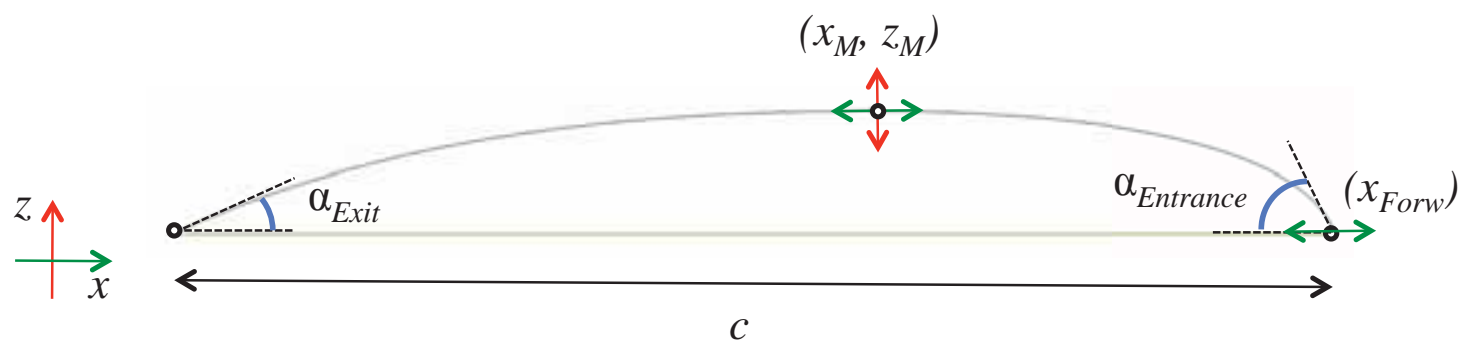

Figure 2: Strut parametric section definition. Allowed movements of points are highlighted as well as possible angle changes.

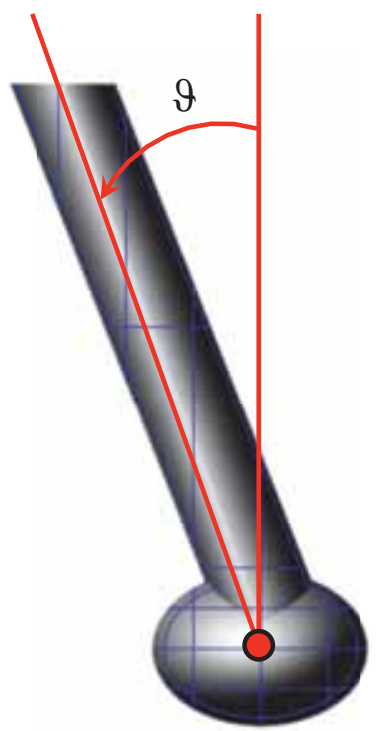

Figure 3: Reference system for the strut rotation by the canting angle, $\vartheta$ 
In total, the global and local shape variations of the struts and of the submerged hulls of the unconventional SWATH depend on by a combination of 23 free parameters. The complete set of free parameters used to create the SWATH shape is listed in Table 2; to define a simple identification system, a progressive name from $X_{1}$ up to $X_{23}$ is associated to the original name of each parameter; moreover, the hull zone on which the parameter acts is identified by a code: $U H$ stands for Underwater Hull, STA means Strut Aft and STF Strut Forward.

Table 2: List of parameters used to generate the SWATH model.

\begin{tabular}{ccc|ccc}
\hline Number & Name & Zone & Number & Name & Zone \\
\hline $\mathrm{X}_{1}$ & $\mathrm{P}_{\mathrm{Z} 1}$ & $\mathrm{UH}$ & $\mathrm{X}_{13}$ & $\alpha_{\text {Exit }}$ & $\mathrm{STA}$ \\
$\mathrm{X}_{2}$ & $\mathrm{P}_{\mathrm{X} 2}$ & $\mathrm{UH}$ & $\mathrm{X}_{14}$ & $X_{\text {Aft }}$ & $\mathrm{STA}$ \\
$\mathrm{X}_{3}$ & $\mathrm{P}_{\mathrm{Z} 2}$ & $\mathrm{UH}$ & $\mathrm{X}_{15}$ & $c$ & $\mathrm{STA}$ \\
$\mathrm{X}_{4}$ & $\mathrm{P}_{\mathrm{X} 3}$ & $\mathrm{UH}$ & $\mathrm{X}_{16}$ & $X_{M}$ & $\mathrm{STF}$ \\
$\mathrm{X}_{5}$ & $\mathrm{P}_{\mathrm{Z} 3}$ & $\mathrm{UH}$ & $\mathrm{X}_{17}$ & $Z_{M}$ & $\mathrm{STF}$ \\
$\mathrm{X}_{6}$ & $\mathrm{P}_{\mathrm{X} 4}$ & $\mathrm{UH}$ & $\mathrm{X}_{18}$ & $\alpha_{\text {Entrance }}$ & $\mathrm{STF}$ \\
$\mathrm{X}_{7}$ & $\mathrm{P}_{\mathrm{Z} 4}$ & $\mathrm{UH}$ & $\mathrm{X}_{19}$ & $\alpha_{\text {Exit }}$ & $\mathrm{STF}$ \\
$\mathrm{X}_{8}$ & $\mathrm{P}_{\mathrm{Z} 5}$ & $\mathrm{UH}$ & $\mathrm{X}_{20}$ & $X_{\text {Forw }}$ & $\mathrm{STF}$ \\
$\mathrm{X}_{9}$ & $\mathrm{~B} / \mathrm{H}$ & $\mathrm{UH}$ & $\mathrm{X}_{21}$ & $c$ & $\mathrm{STF}$ \\
$\mathrm{X}_{10}$ & $X_{M}$ & $\mathrm{STA}$ & $\mathrm{X}_{22}$ & $Y_{\text {Transl }}$ & STA/STF \\
$\mathrm{X}_{11}$ & $Z_{M}$ & $\mathrm{STA}$ & $\mathrm{X}_{23}$ & $\vartheta$ & $\mathrm{STA} / \mathrm{STF}$ \\
$\mathrm{X}_{12}$ & $\alpha_{\text {Entrance }}$ & $\mathrm{STA}$ & & & \\
\hline
\end{tabular}

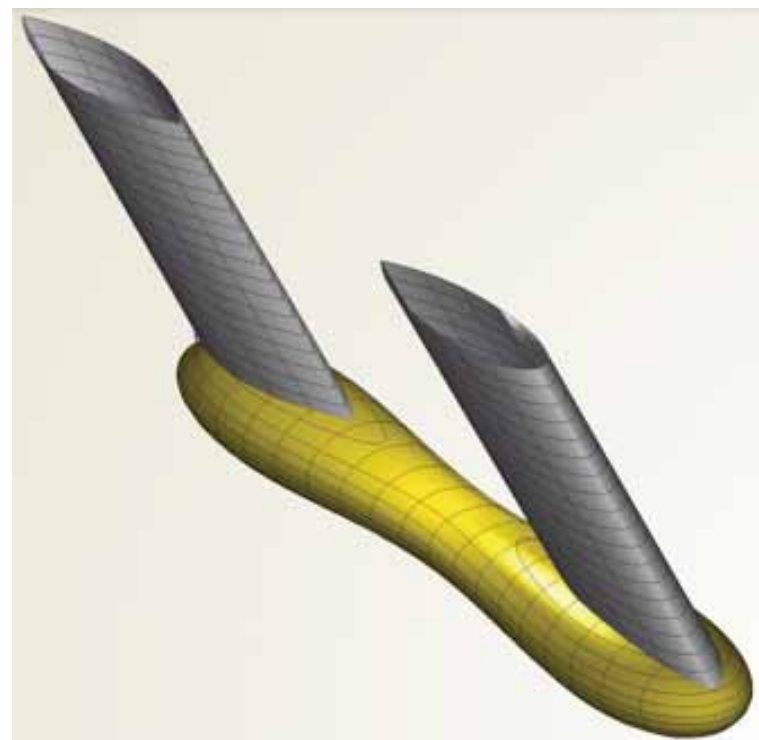

Figure 4: Initial hull shape. Ellipticity factor $\mathrm{f}=1.4$

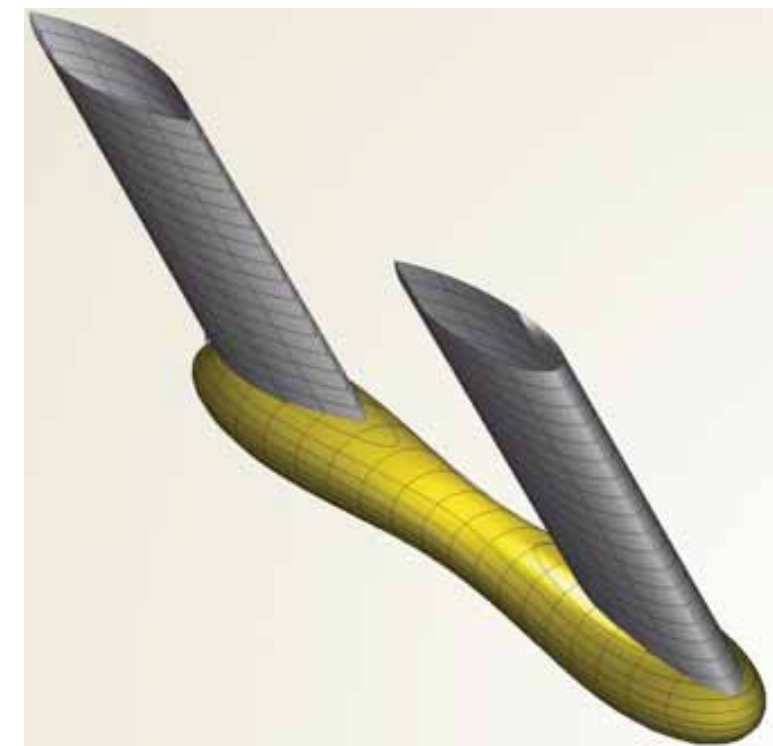

Figure 5: Ellipticity factor variation $(\mathrm{f}=1.2)$ 


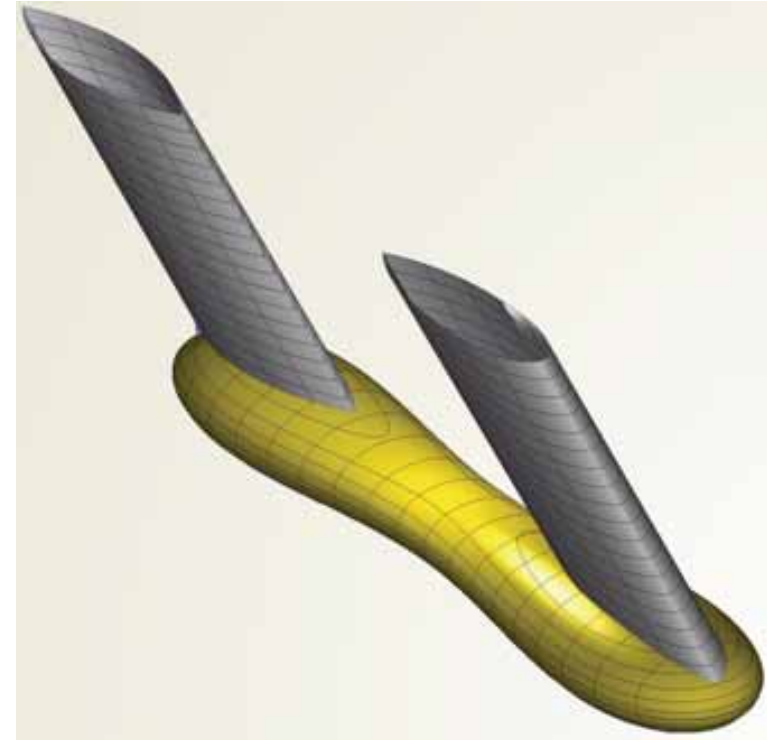

Figure 6: Ellipticity factor variation $(\mathrm{f}=1.6)$

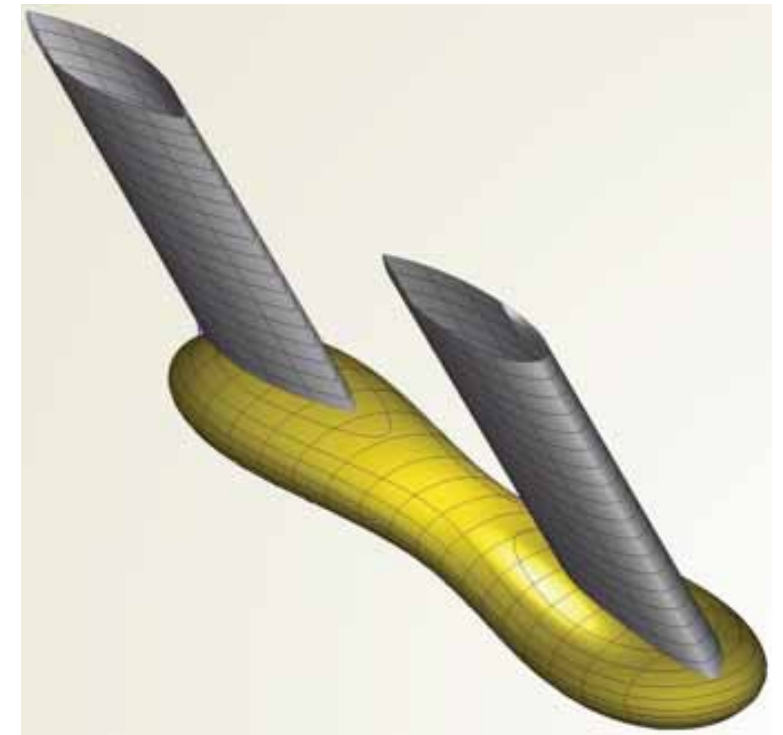

Figure 7: Ellipticity factor variation ( $\mathrm{f}=1.8)$

Some examples of shape variations are presented in the following ignoring the feasibility of the design with respect to the optimization constraints. Three hull shape variations, obtained by a change of the ellipticity factor, are shown in Figure 4 to Figure 7 together the initial design (demi-hull); the bow on the right in all figures. Increasing the value of such a parameter, while keeping fixed the height, the beam of the underwater hull is increased. A change in the ellipticity factor alone can cause a relevant variation of the volume that needs to be met during optimization while the longitudinal center of gravity of the hull is not modified. This is one of the main parameters that the algorithms plays on to cope with global changes of the underwater body shape: the transverse sections can change from an ellipsis with vertical main axis to a section with the same area but with horizontal main axis, passing through a perfect circular section.

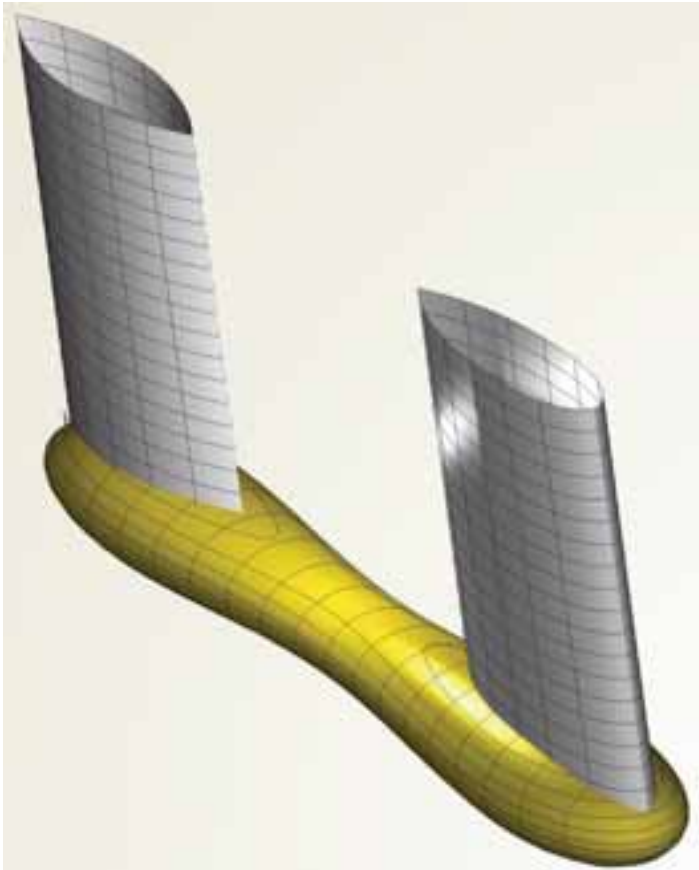

Figure 8: Upright struts (null canted angle)

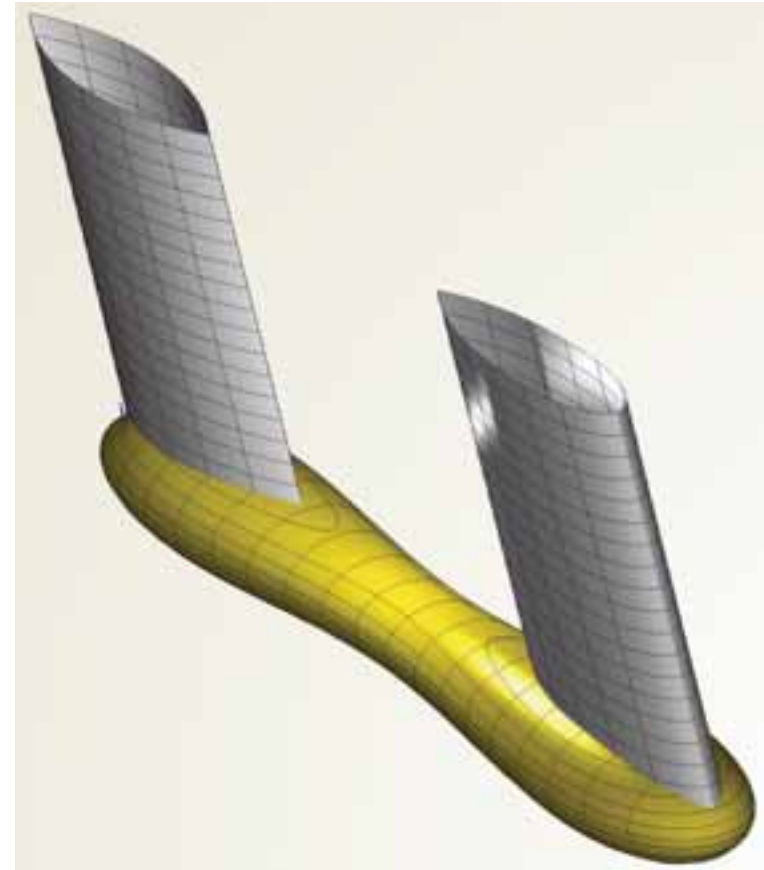

Figure 9: Increased struts canted angle $\left(\vartheta=10^{\circ}\right)$ 


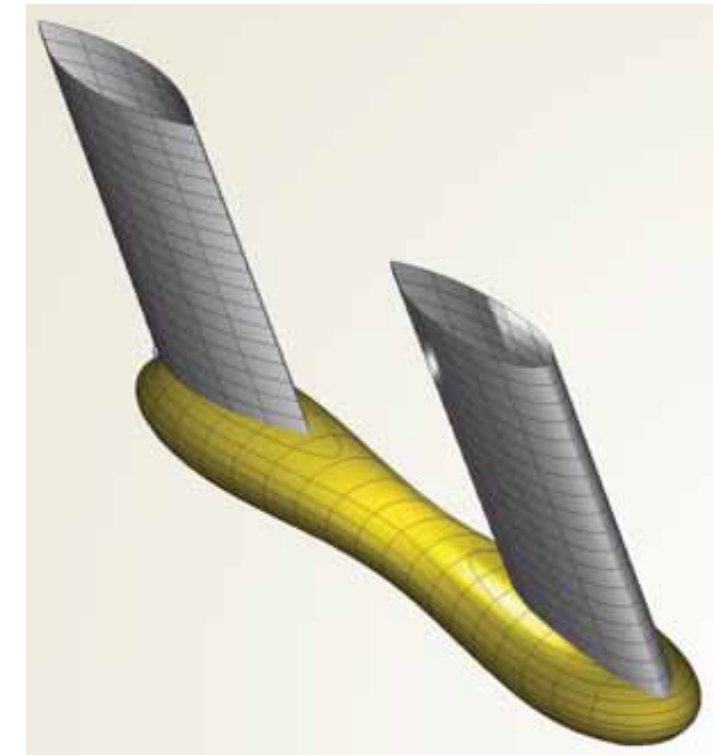

Figure 10: Increased struts canted angle $\left(\vartheta=20^{\circ}\right)$

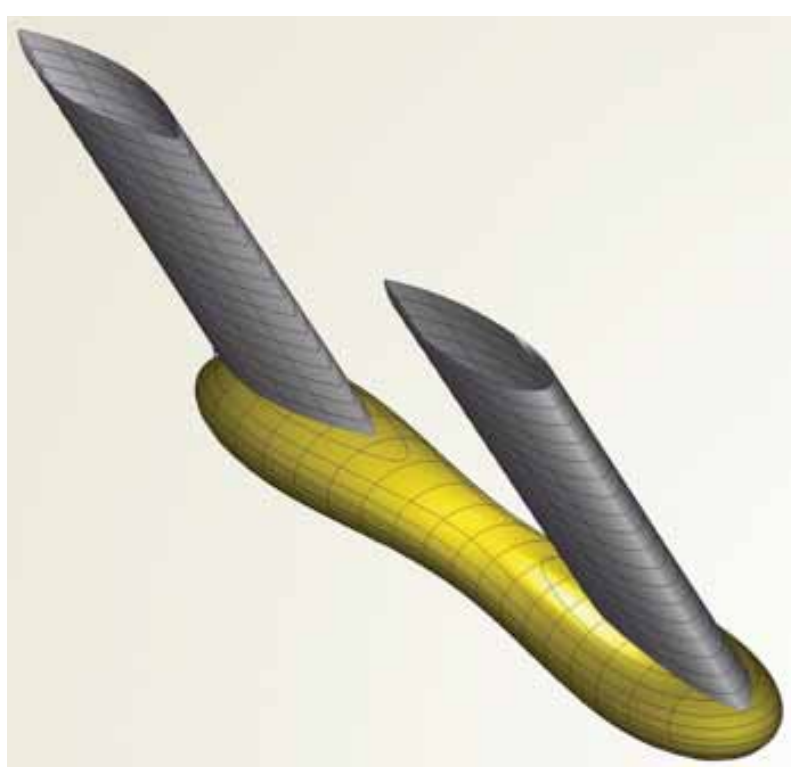

Figure 11: Increased struts canted angle $\left(\vartheta=30^{\circ}\right)$

Another relevant shape variation consists in the canting angle of the struts; this is achieved by changing a single parameter (the angle $\theta$ in Figure 3). In Figure 8 to Figure 11 four SWATH shape variants are presented (one demi-hull only) where the canted angle is increased from $0^{\circ}$ up to $30^{\circ}$. In principle, the distance between the two demi-hulls should be modified to follow the struts canting angle; this will cause, in turn, the adjustment of some parameters of the overall SWATH design, such as for example the width of the deck or at least the position of the strut-deck joint; also, the draught of the vessel should be adjusted on the new strut canted angle. In the optimization study presented the following hypothesis are made: the general design of the vessel (and with it its deck area) has been unvaried; the draught was kept for operating reasons; the potential effects of a variation of the strut canting angle on the general arrangements and on the vessel's draught have been neglected in this study which primarily concerns on finding relations between the shape and the drag characteristics of canted struts SWATH geometries.

The last parametric shape variation considered involves the local shape of the forward strut. The forward strut entrance angle, measured at the leading edge in the strut xy-plane can vary from $30^{\circ}$ up to $90^{\circ}$; an example of two geometries is shown in Figure 12 and 13. As mentioned before all the changes are applicable to both forward and aft struts independently. Moreover, since the plane sections of the struts are kept, this change is the same all over the height the strut. 


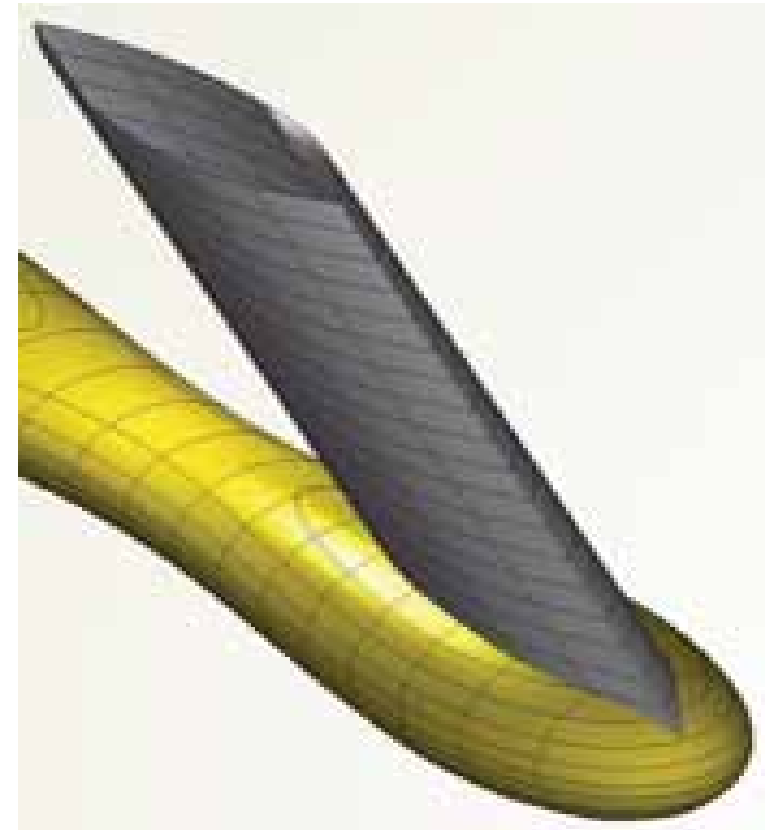

Figure 12: Forward strut entrance angle $\left(\alpha=30^{\circ}\right)$

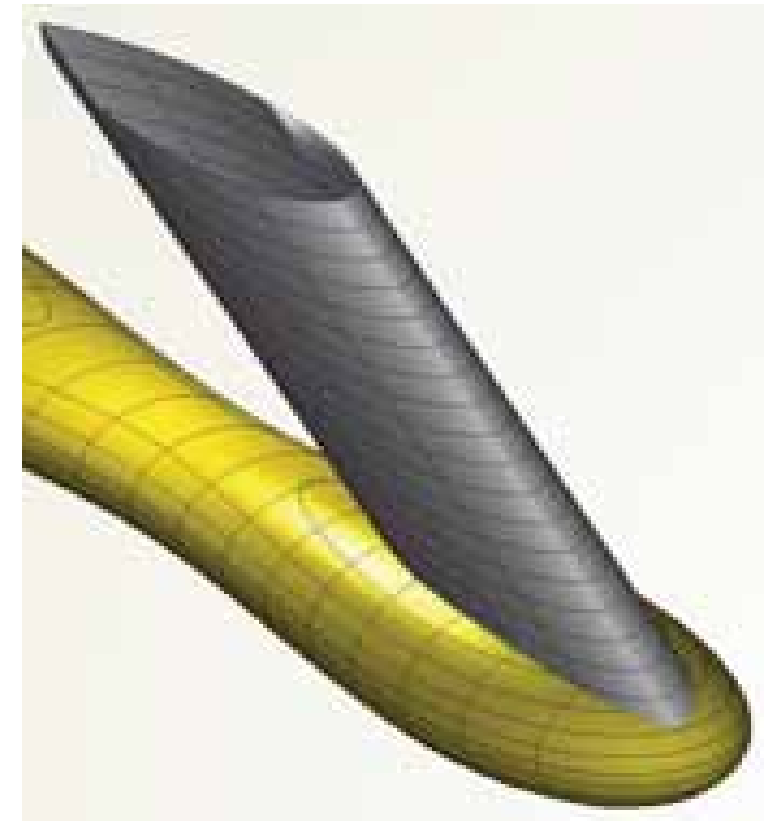

Figure 13: Forward strut entrance angle $\left(\alpha=70^{\circ}\right)$

\section{PREDICTION OF THE HYDRODYNAMIC RESISTANCE IN CALM WATER BY BOUNDARY ELEMENT METHOD}

The wave making resistance of the SWATH is computed by means of a first order linear Boundary Element Method (BEM) that uses Rankine sources distribution on the hull and on the free surface to solve for the potential flow in the near field. The boundary value problem is formulated in terms of total velocity potential, $\Phi=U_{\infty} x+\phi$, and perturbation potential, $\phi$, both satisfying the Laplace Equation (1a). The problem is completed by a suitable set of boundary conditions, namely the Neumann type condition over the wetted hull surface (1b), the kinematic (1c) and dynamic (1d) conditions on the free surface, $\zeta=\zeta(x, y)$, both linearized around the double-model flow, and the radiation condition (1e) for the upstream disturbance. The boundary value problem for wave resistance computation is then written as:

$$
\begin{cases}\Delta \Phi=0, \Delta \phi=0 & \\ \nabla \Phi \cdot n=0 & \text { on the hull surface } \\ \nabla \Phi \cdot \nabla \zeta=0 & \text { on the free surface } \\ g \zeta+1 / 2 \nabla \Phi \cdot \nabla \Phi=1 / 2 U_{\infty} & \text { on the free surface } \\ \phi \rightarrow 0 & x \rightarrow \infty\end{cases}
$$

The free surface kinematic and dynamic boundary conditions are linearized around the double body flow (Bruzzone, 1994). The domain, namely the hull and the free surface, is discretized by quadrangular elements; constant distribution of Rankine sources is used over each panel. Derivatives of the induction coefficients are approximated with a 4-point discrete operator, both in the longitudinal and transversal direction. Wave resistance is found by integrating the potential flow pressure over the hull-wetted surface. Lift effects are neglected in the present formulation of the method; even if the struts are inclined in a transversal plane by canting angle, this approximation is considered acceptable (in steady conditions) since they are designed with symmetric profiles having zero angle of attack with respect to the undisturbed free stream.

The BEM was validated in case of different fast mono- and multi-hulls (Brizzolara et al., 1998), including unconventional single-strut SWATHs (Brizzolara, 2004, Bruzzone \& Brizzolara, 2007 or Brizzolara et al., 
2015) from which the results of the numeric-experimental validation study of the total resistance coefficient are reported in Figure 14. The total resistance is very well approximated by the present BEM with viscous corrections in a large range of Froude numbers, starting from Fn 0.25 up to Fn 0.9.

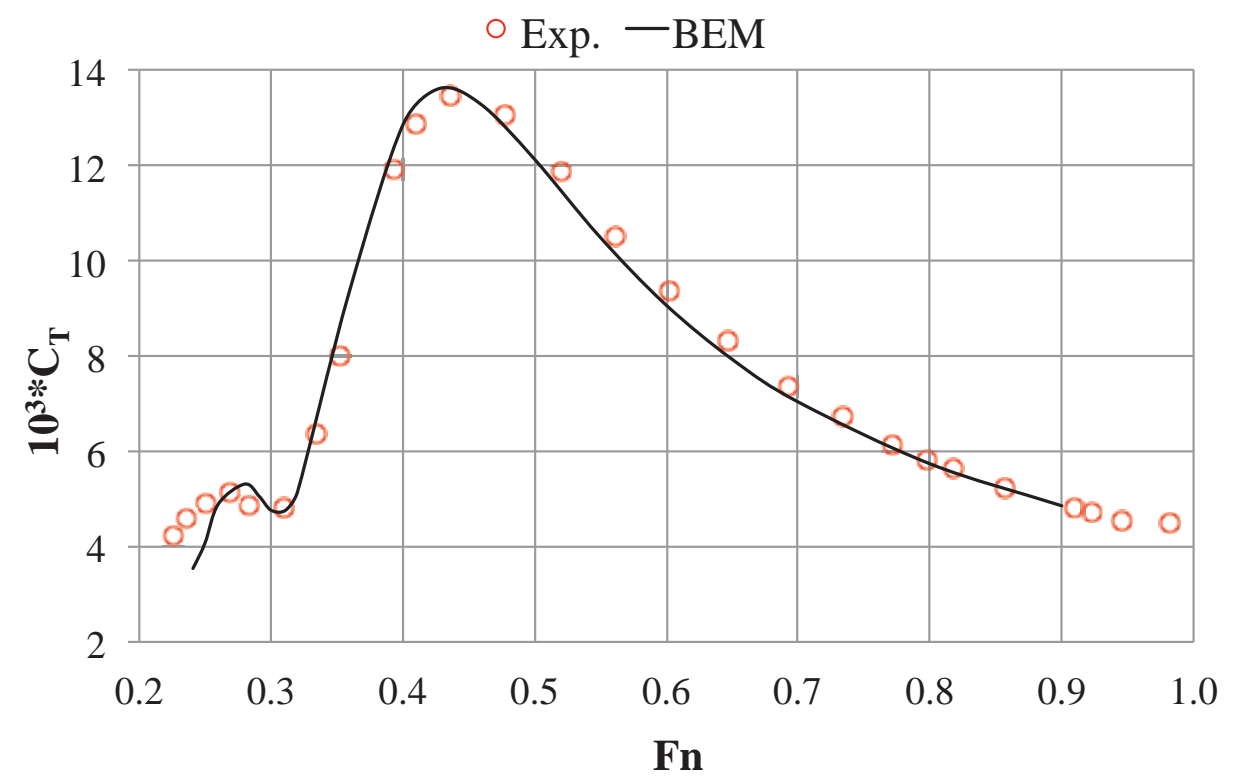

Figure 14: Numeric-Experimental comparison of the total resistance coefficient for a single-strut SWATH

The total resistance $R_{T}$, is calculated summing up the wave-making resistance $R_{W}$, and the viscous resistance, $\mathrm{R}_{\mathrm{V}}$. The latter is computed in this study by the ITTC' 57 formulation, corrected with a form factor, $k=0.2$, deduced from the solution of the thin boundary layer around similar unconventional hull shapes (Brizzolara, 2004). The change of the form factor due to the variation of the hull shape is neglected here, main concern being on wave resistance reduction due to wave cancellation effects at the considered Froude numbers. Hence the viscous resistance, for this study, depends on a hull shape variation only through the different wetted surfaces.

The final configuration of the computational domain, i.e. the number and distribution of the mesh elements, is chosen as result of a basic sensitivity analysis; the variation of the value of the resistance due to mesh refinement is monitored until convergence is reached. An example of free surface mesh sensitivity analysis is shown in Figure 15: a progressive variation of two free surface parameters that regulate the number of elements on the free surface along the longitudinal and transversal direction is used. The variation of the resistance $\delta\left(R_{T}\right)$, plot in the graph as a function of the total number of elements on the free surface, $n_{F S_{-} E l e m}$, is relative to the minimum value reached at convergence.

The final computational grid consists of 4676 total elements, 1824 of which are used to discretize the hull and the remaining 2852 represent the free surface. An example of the hull and free surface panel meshes is shown in Figure 16. The spacing of the surface elements follows a geometric progression both in the lateral direction in the region outside the demi-hull and in the longitudinal direction behind the stern of the aft strut while they are uniformly distributed between the symmetry plane and the inner side of the demi-hull. The overall domain size is chosen so that no waves are reflected by the side boundary and no appreciable influence is given by the proximity of the upstream and downstream boundaries. 


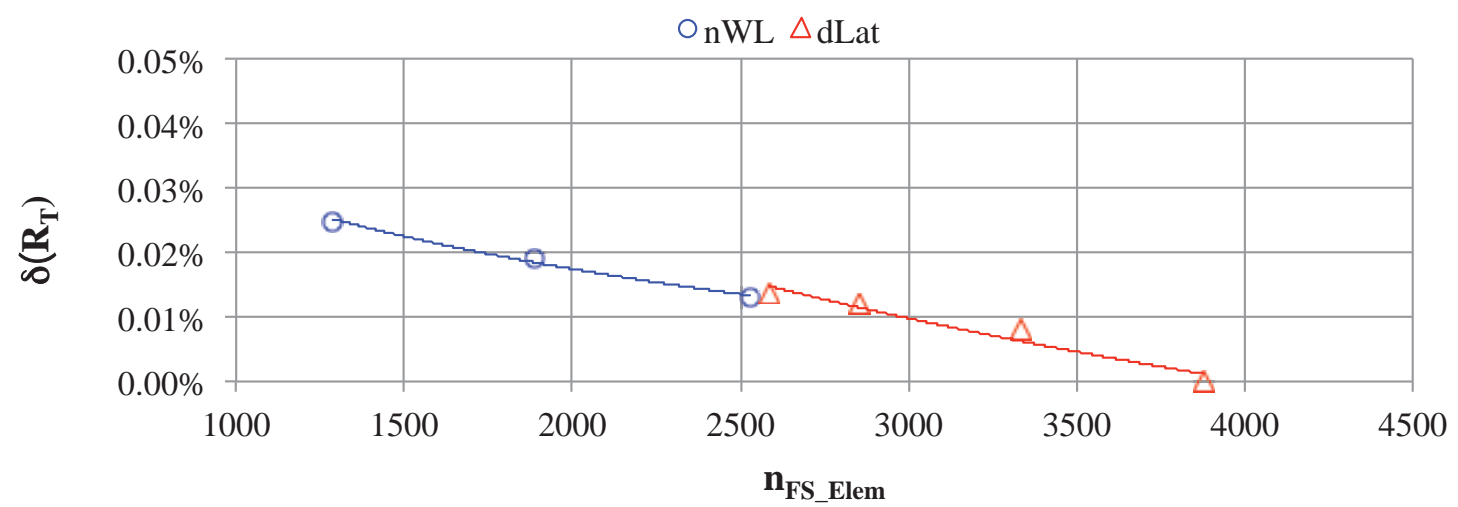

Figure 15: Example of free surface panel mesh sensitivity. Resistance relative variation is monitored with respect to changes in the number of waterline element and lateral dimension of the first element close to the hull.

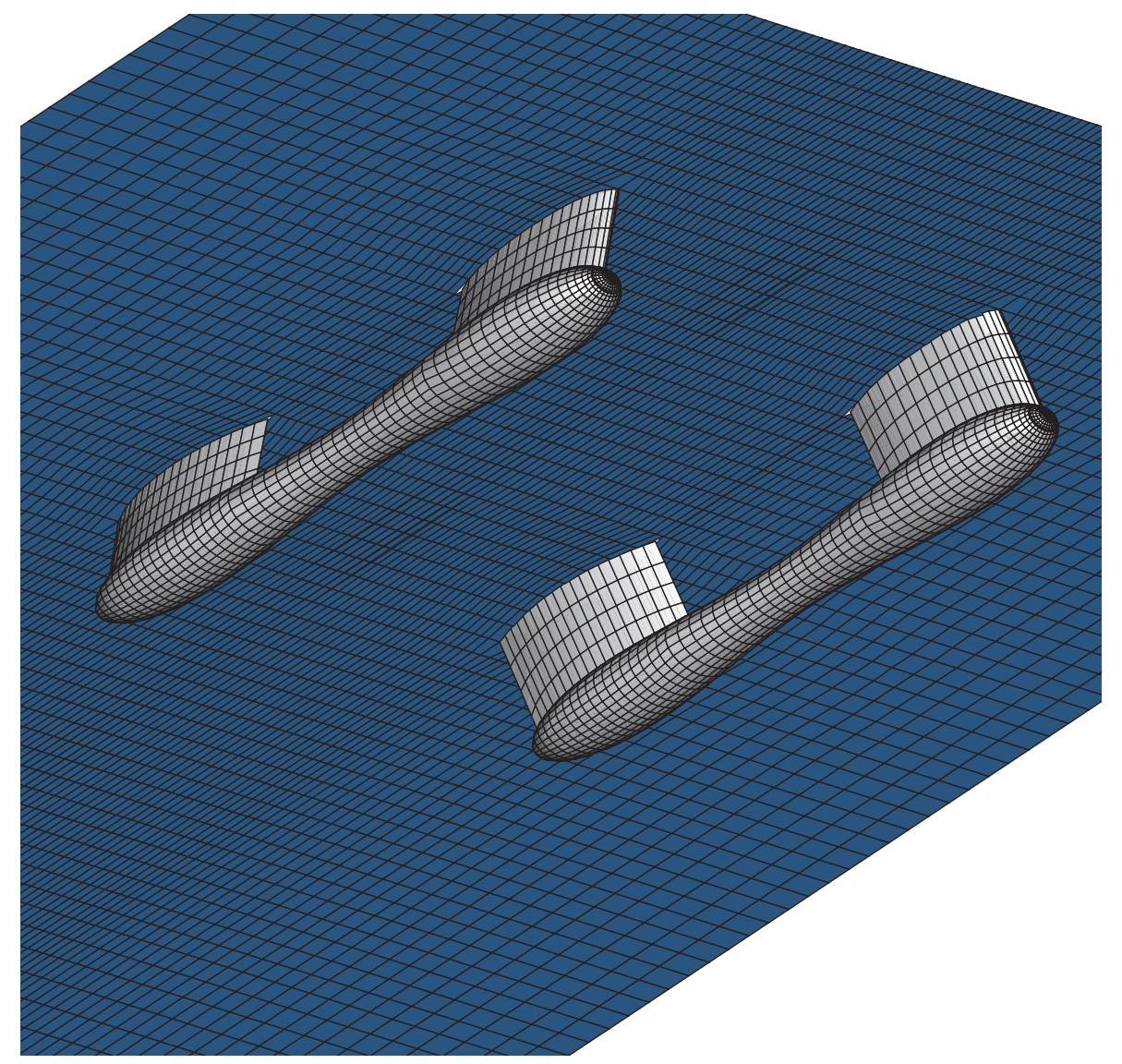

Figure 16: Extract of the final hull and free surface panel mesh configuration

\section{NUMERICAL OPTIMIZATION STUDY TO ACHIEVE THE OPTIMUM CANTED STRUTS ARRANGEMENT FOR UNCEONVENTIONAL SWATH}

To analyze the effect of the canted strut arrangement on the hydrodynamic calm water resistance performance of the proposed SWATH design, three optimization cases have been carried out:

1. Underwater hull alone;

2. Underwater hull + struts;

3. Underwater hull + struts at two different speeds. 
The first and the second processes are single objective optimizations aimed at reducing the total resistance in calm water at cruise speed, corresponding to $\mathrm{Fn}=0.6$. The first is meant to optimize the underwater hull shape alone, keeping the struts shape of the reference design; in the second case, the shape of both struts is fully free to change together to the main hulls. The last run, instead, is a multi-objective full parametric optimization aimed to minimize the total resistance in calm water of the complete hull at two different speeds, corresponding to $\mathrm{Fn}=0.6$ and $\mathrm{Fn}=0.4$.

Recalling the definitions of Table 2, the first single objective optimization case is based on parameters $\mathrm{X}_{1}$ to $\mathrm{X}_{9}$; the other two computations, in addition to the previous ones, can perform variations on parameters $\mathrm{X}_{10}$ to $\mathrm{X}_{23}$ too.

The objective function used for all optimization runs presented in the paper is the relative reduction of the total resistance over displacement $\left(\mathrm{R}_{\mathrm{T}} / \Delta\right)$ with respect the reference hull form, hence:

$F_{\text {Obj }}=\frac{R_{T} /\left.\Delta\right|_{I D}}{R_{T} /\left.\Delta\right|_{\text {Init }}}$

It follows that the initial (reference) design has $F_{O b j}=1$. So the total resistance is normalized with the displacement to account for the (small) displacement variations allowed in the various evaluated alternatives (Biliotti et al., 2011). The objective function so defined is then normalized by its value on the reference hull form; this is necessary especially in case of multi-objective optimization, in order to avoid preferences for any of the objectives.

With respect to previous optimization studies (Brizzolara \& Vernengo, 2011), a different set of constraints is used here. 5\% change for volume and longitudinal position of the center of gravity is allowed with respect to the initial design values. The variation of transversal metacentric height is restrained in a $3 \%$ interval around the reference value. Moreover two constraints dealing with internal arrangements are considered: the minimum beam and the minimum height of the station located at the $0.13 \mathrm{~L}_{\mathrm{BP}}$ (from the stern); this is where the electric engines are placed in design of the vessel (Brizzolara et al. 2011). The last two constrains are the maximum thickness of the strut, again to be considered in a relative way with respect to the initial ones. A summary of the constraints definition with the corresponding reference values is given in Table 3.

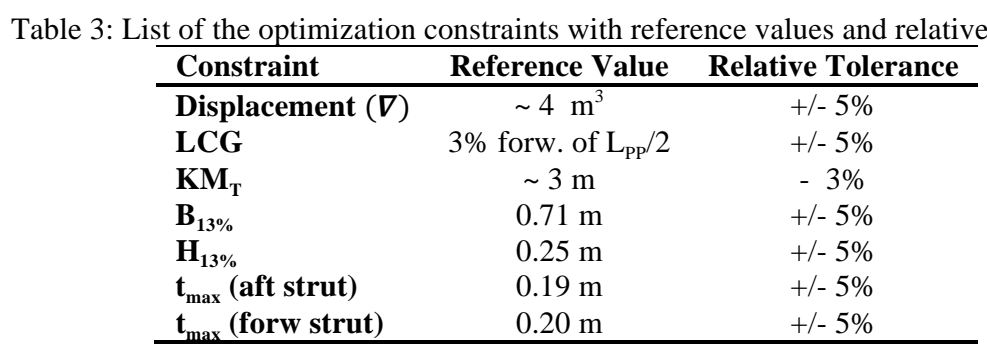

The Non-dominant Sorting Genetic Algorithm II (Deb et al., 2000) is used to drive the search towards the optimum; this is a well-established modern genetic algorithm with enhanced properties of fast and global convergence. It is a population-based algorithm that can handle multiple constrained objective functions concentrating the evaluation on the Pareto frontier of the specific problem. The initial Design of Experiments (DoE), made of 300 designs, is generated as combination of two methods: a first series of designs is created by varying each parameter at a time from the baseline design, to cover the whole allowed variation range; a second set, in order to improve the diversity of the initial population, is created applying the Sobol sequence (Sobol, 
1967) to all parameters. The optimization cases are set up using modeFrontier optimization framework (Esteco, 2014) that simplifies a lot the definition of the automatic procedure and post-processing of the results.

\subsection{Single-objective optimizations: effect of struts shape}

The convergence history of the objective function for the first single-objective optimization, i.e. without any strut shape modifications, is shown in Figure 17. A clear convergence trend is found, in other terms, the spread in the designs performance shrinks as the number of evaluations increases and the moving average value is monotonically decreasing. The maximum reduction of the objective function, obtained at convergence, is $-19 \%$ with respect to the reference initial value $(\mathrm{Obj}$. Function $=1$ corresponds to the total resistance of the reference hull design).

The optimization history of the second single-objective optimization, that is performed accounting for strut shape changes, appears in Figure 18. In the second run, the objective function evaluated by the evolutionary algorithm shows a larger initial spread. This is a direct consequence of the increased number of free parameters. Despite the large number of free parameters (25 in total) the total number of design evaluations needed to reach convergence is almost the same as in the previous case. There is still a sharp convergence trend on a minimum value that amounts to $-29 \%$ of the initial value. Comparing the two results suggests that the effect of considering the struts geometry into the parametric optimization accounts for about $10 \%$ of the total resistance reduction. So, as initially expected, the geometry of the struts plays a role in resistance reduction, although of minor importance with respect to the underwater hulls, that account for the $2 / 3$ of the total reduction.

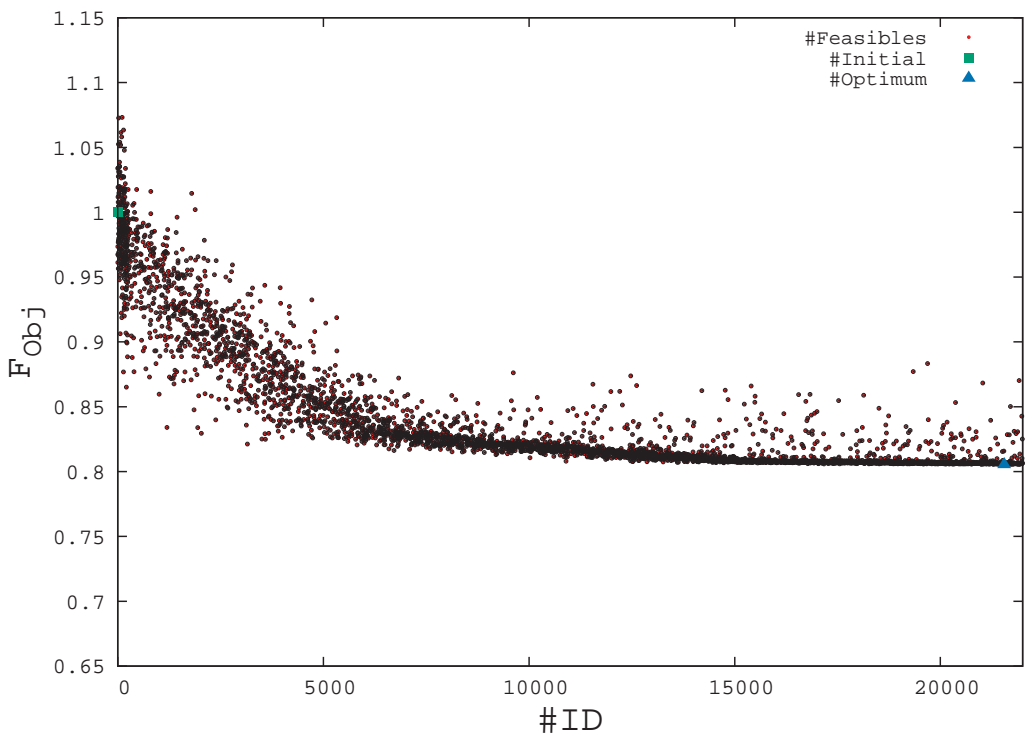

Figure 17: Optimization history. Single-Objective run. Fixed struts shapes 


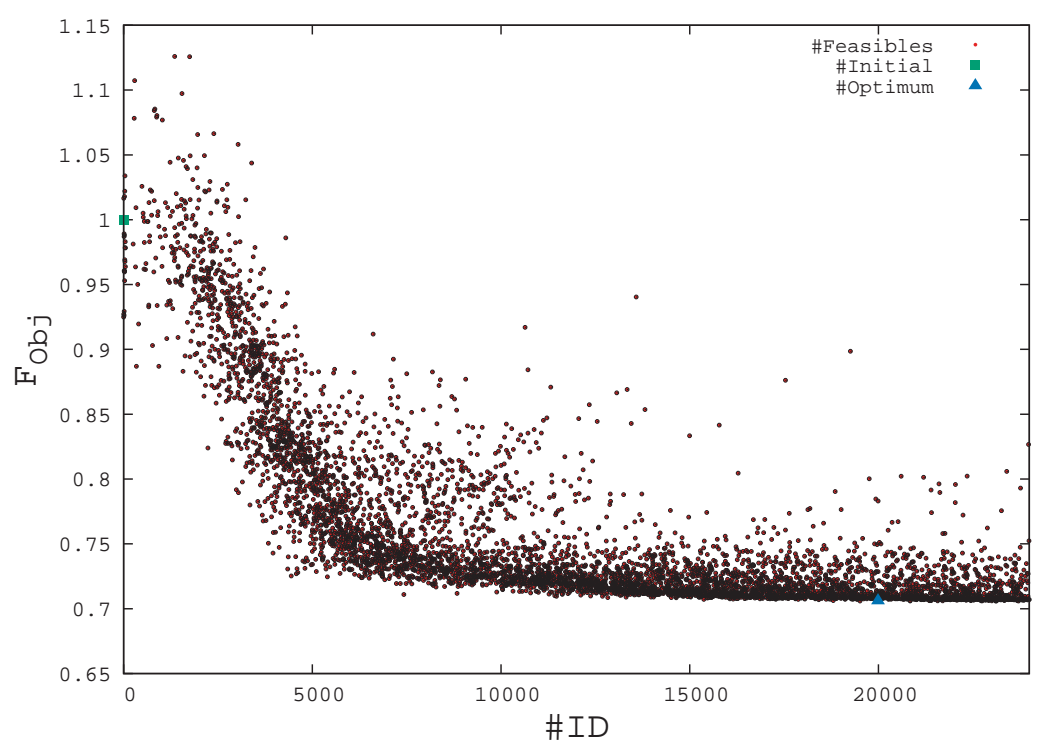

Figure 18: Optimization history. Single-Objective run. Struts shapes free to change

The two computations have been run in parallel on 32 cores for about two days. The interesting results described, justified a further study on the effect of multiple operating speeds, which is presented in the next subsection.

The perspective view of the shape of the initial SWATH (demi-hull) is presented in Figure 19 and it is compared to the optimum shapes obtained from the $1^{\text {st }}$ and the $2^{\text {nd }}$ optimizations in Figure 20 and Figure 21 , respectively. The bow points to the left of the perspective view. The same stations, buttocks and waterlines are represented to facilitate the shape comparison. In both cases part of the volume of the underwater body has been shifted forward creating a more cylindrical shape in the rear portion. The bow of the second optimum hull (Figure 21) shows a finer shape than the first optimum hull. This is probably due to the adaptation of the underwater shape to the different wave trains generation by the struts whose canting angle is decreased by the optimization algorithm in favor of a more vertical configuration.
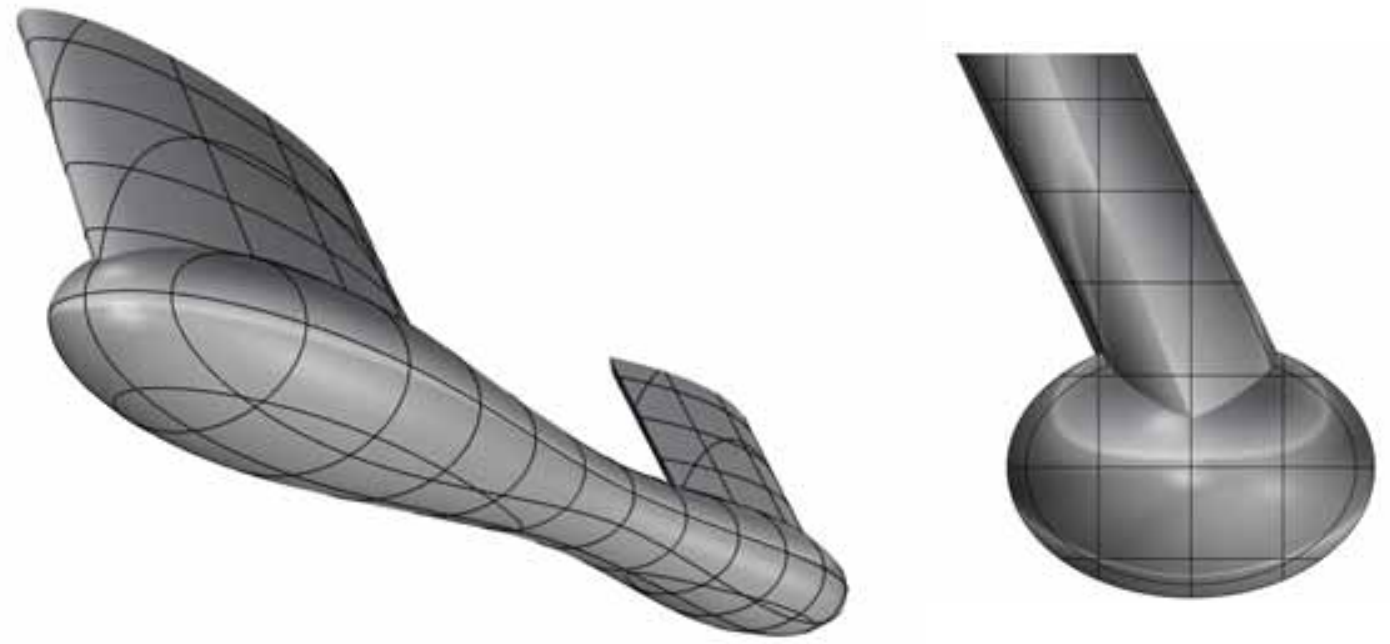

Figure 19: Perspective/front view of the initial (reference) hull shape (only one demi-hull is presented) 

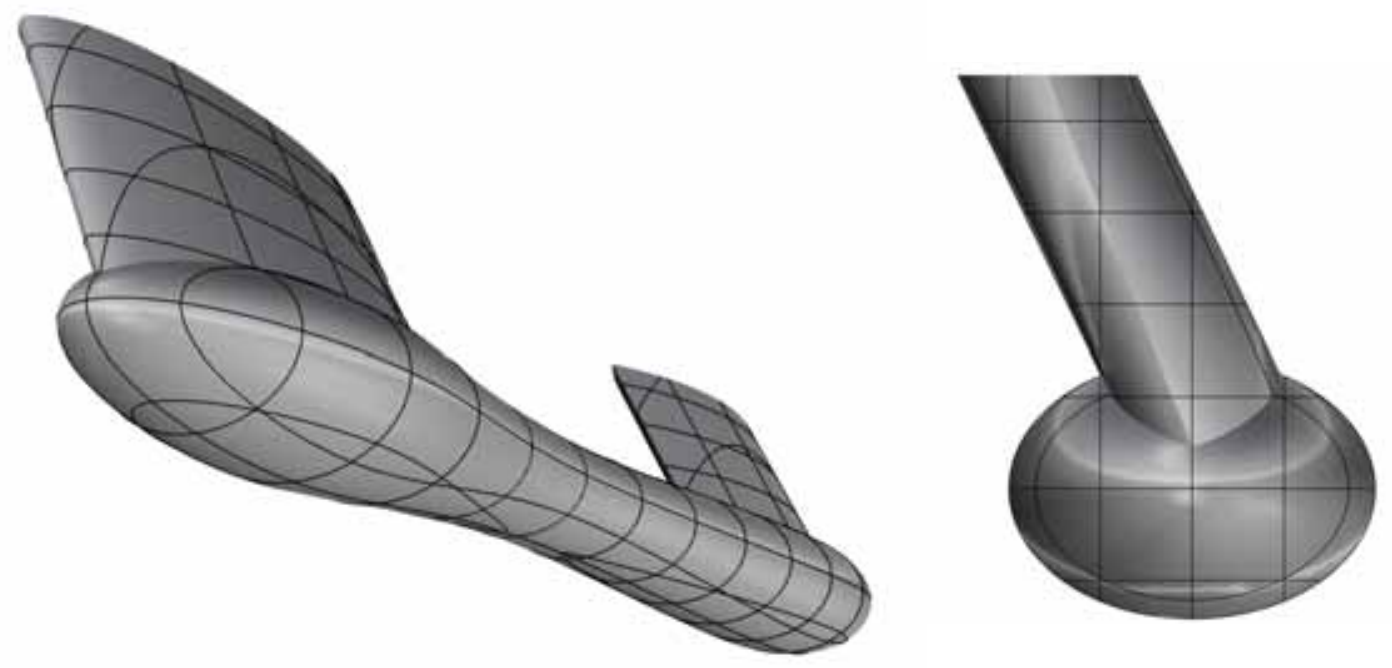

Figure 20: Perspective/front view of the optimized hull from the $1^{\text {st }}$ run: free underwater hull shape, fixed struts. (only one demi-hull is represented).
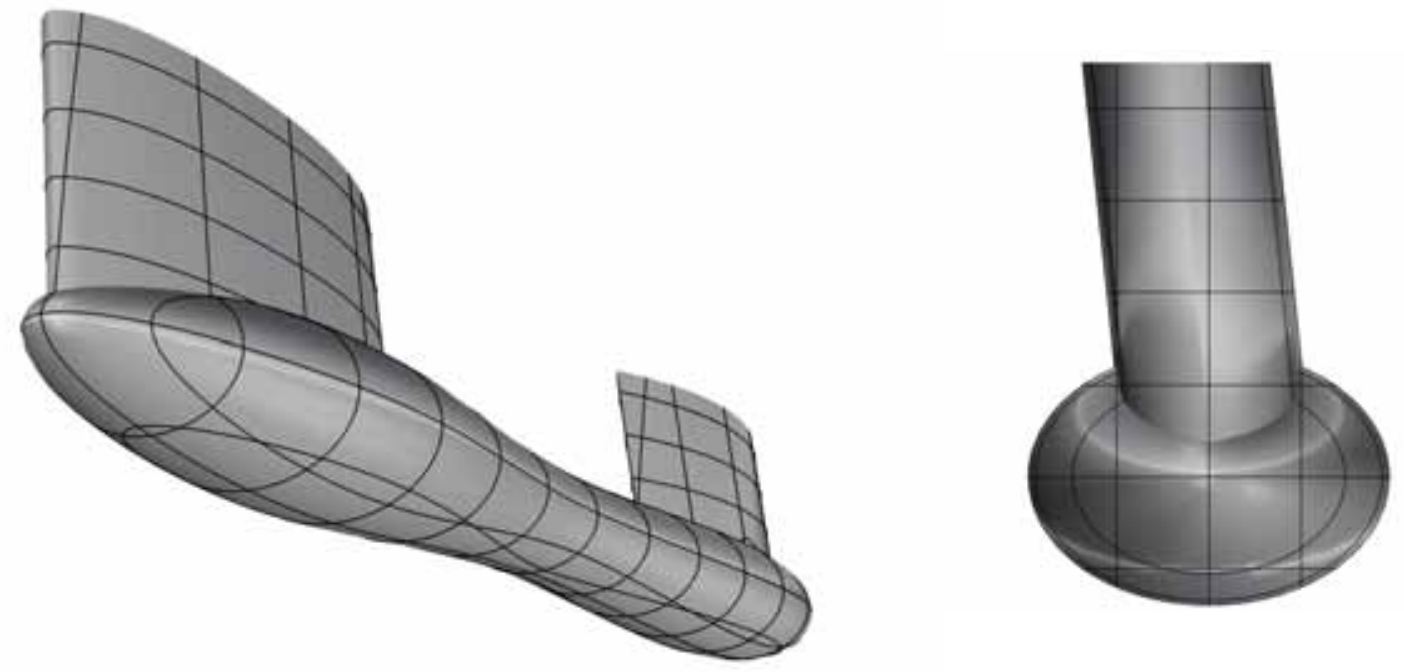

Figure 21: Perspective/front view of the optimized hull from the $2^{\text {nd }}$ run: free twin struts shape and canting angle in addition to the underwater hull form (only one demi-hull is represented).

The free wave patterns of the initial hull and those of the first two optimized hulls are compared in Figure 22 to Figure 24 respectively. At this relatively high Froude number the wave energy is concentrated in the divergent wave systems and the major components are created at the leading and trailing edge of the two struts. The variation of the shape of the underwater hull (especially around its intermediate restricted section) is able to modify the amplitudes of the divergent waves. The second optimum appears to create higher waves close to the struts waterlines, which in fact generate a wave interference effect that shows lower resulting waves in between the two demi-hulls.

The major portion of the wave resistance derives from the integration of the dynamic pressure on just the underwater hulls surfaces and not on the struts. So in case of the first optimization (struts fixed), it is natural that the shape of the underwater hull may adapt, during the evolution, in order to minimize the pressure drag deriving from the divergent wave field generated by the struts. In case of the second optimization, instead, the struts can adapt their shape to minimize the generation of the divergent wave trains that, combined with the hull shape, gives the lowest pressure drag. 


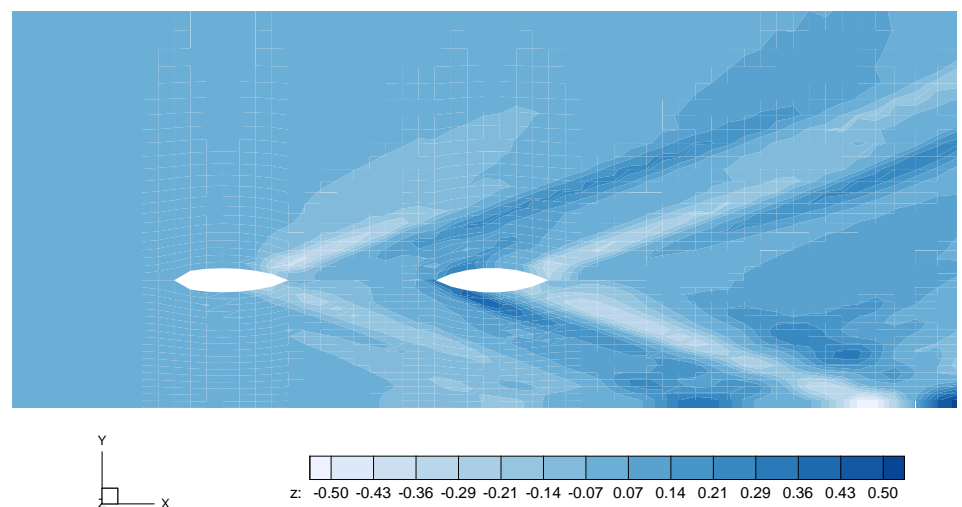

Figure 22: Wave pattern of the reference hull form $(\mathrm{Fn}=0.6)$

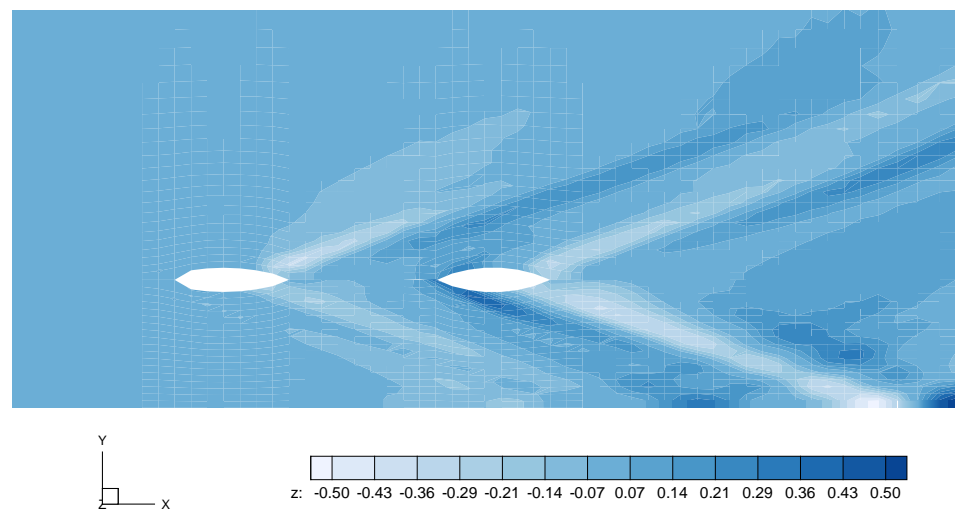

Figure 23: Wave pattern of the optimized design of the $1^{\text {st }}$ run $(\mathrm{Fn}=0.6)$

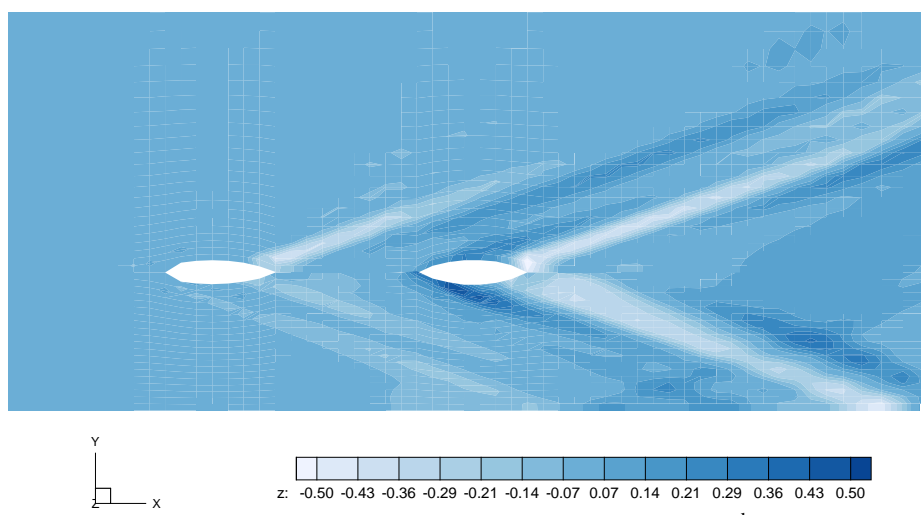

Figure 24: Wave pattern of the optimized design of the $2^{\text {nd }}$ run $(\mathrm{Fn}=0.6)$

\subsection{Multi-objective hydrodynamic shape optimization results}

The objective of this optimization study is the reduction of the total calm water resistance at two different operating speeds: cruise speed $\mathrm{Fn}=0.6$ and transfer mode (lower speed) corresponding to Fn=0.4; the latter corresponds to a hump in the wave resistance was noted in the previous single speed optimization study (high speed only). All parameters defining the shape of the underwater hull and of the two struts are included in the in this new multi-objective optimization, as they were considered in the second single-objective optimization, previously described. The ensemble of all feasible designs obtained is shown in Figure 25 and in a restricted region close to the optimum designs in Figure 26. Feasible designs are shown by red dots; the initial design, 
corresponding to the point $(1.0,1.0)$, is highlighted by a green square; the best design for the higher speed is shown as a blue triangle while the best design for the lowest speed is shown as a pink triangle; the best Pareto design, i.e. the best trade-off between the two objectives, is shown with a red rhombus. A very satisfactory convergence has been achieved after 60000 designs (including unfeasible). The computation was run on a 32 cores workstation for about seven days. The best Pareto design achieves a resistance reduction of $36 \%$ at low speed and of $30 \%$ at high speed. The improvement obtained at the higher speed is consistent with that achieved during the second optimization process at the same speed.

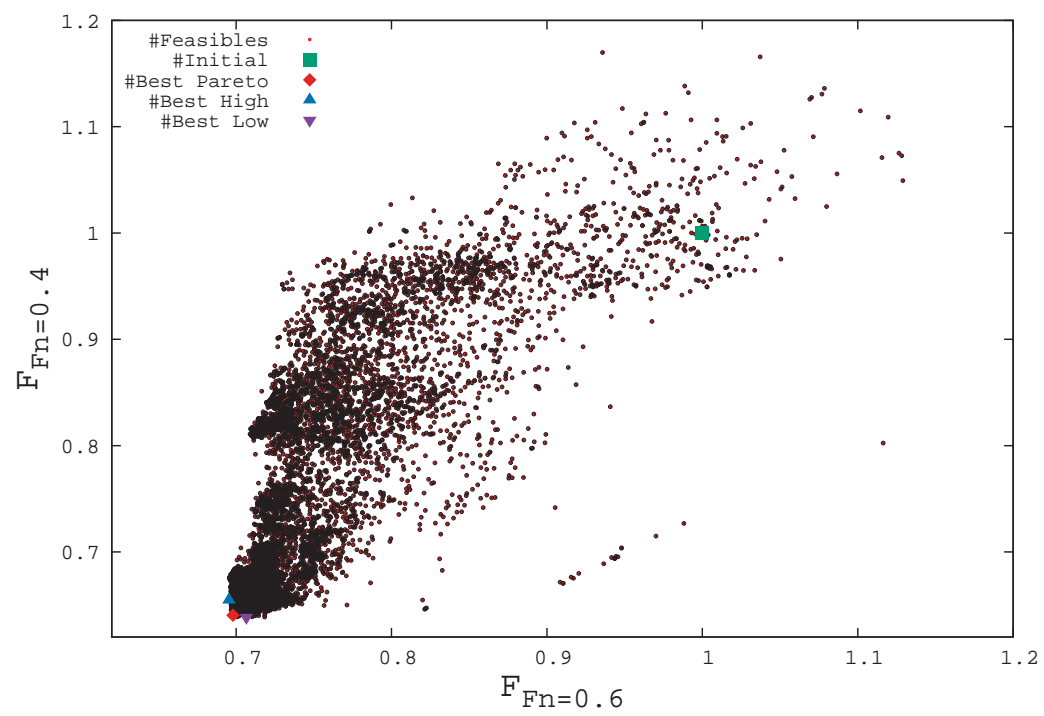

Figure 25: Multi-objective function scatter diagram with all feasible designs. Highlighted: Initial design (green square). Best Pareto design (red rhombus). Best design for $F n=0.6$ (blue triangle). Best design for $F n=0.4$ (pink triangle).

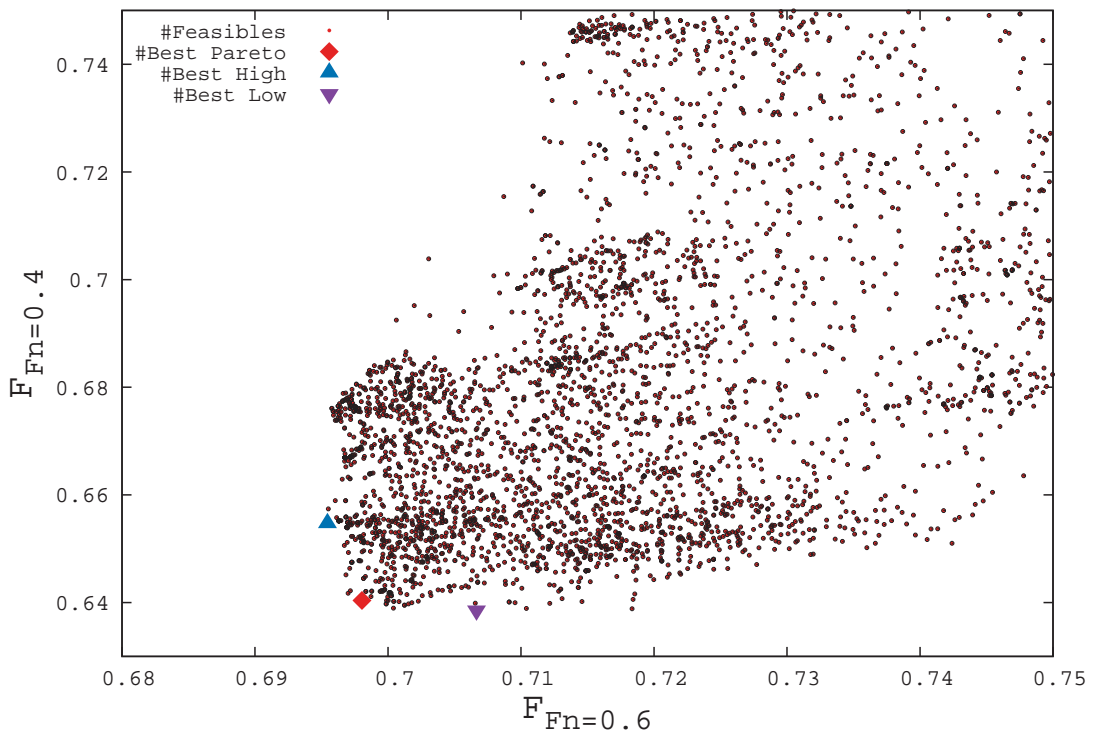

Figure 26: Zoom of the multi-objective function scatter diagram with all feasible designs close to the Pareto Frontier. Highlighted: Initial design (green square). Best Pareto design (red rhombus). Best design for Fn=0.6 (blue triangle). Best design for Fn=0.4 (pink triangle).

The perspective view of the Best Pareto design is shown in Figure 27. Compared to the shape obtained in the previous optimization studies, this hull has almost vertical struts while the shape of the underwater body is very close to the one optimized in the $2^{\text {nd }}$ single-objective run but the maximum breath of the forward hull hump is slightly shifted backwards, creating an even streamlined forward body shape.

The wave pattern comparison between the initial design and the Best Pareto at the highest and lowest speeds are 
shown in Figure 28 and Figure 29, respectively. There are some differences in the waves generated in proximity of the struts, caused by the variations in the local shape of the two hulls. The major effect seen from the wave patterns is a different interference that modifies the free waves pattern in between the hulls. Also the waves in the free surface aside the hulls seem to be less scattered.
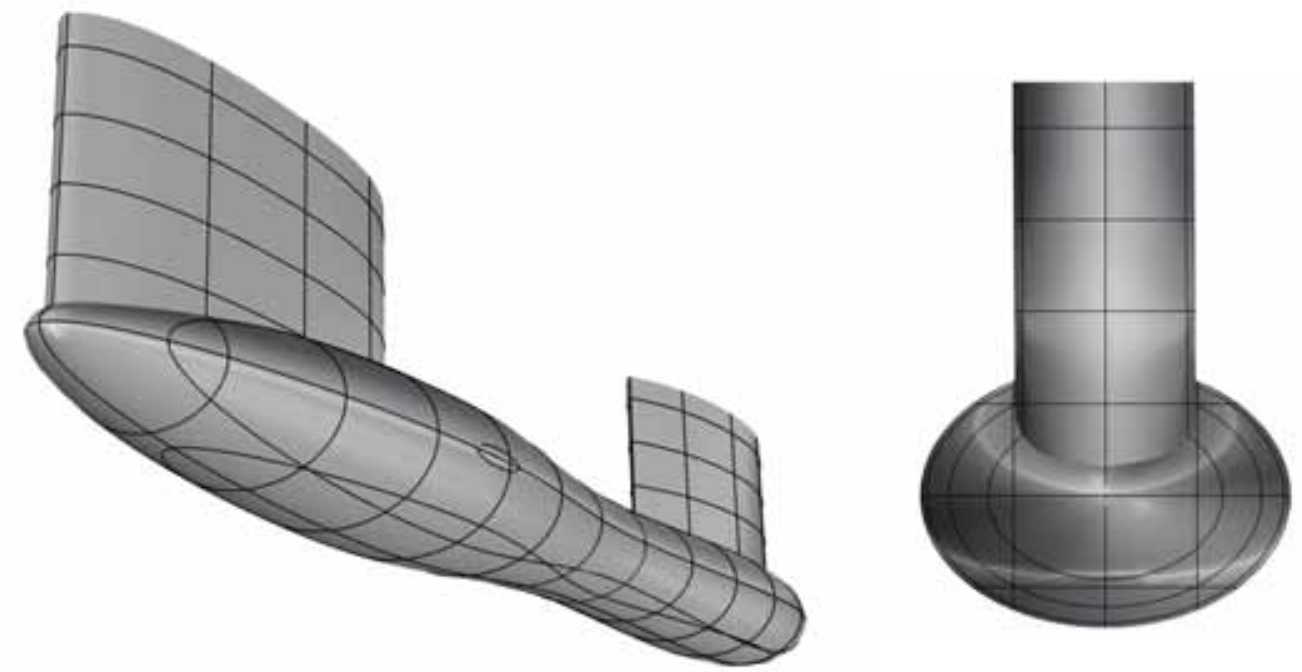

Figure 27: Perspective view of the Best Pareto hull from the multi-objective run (demi-hull).

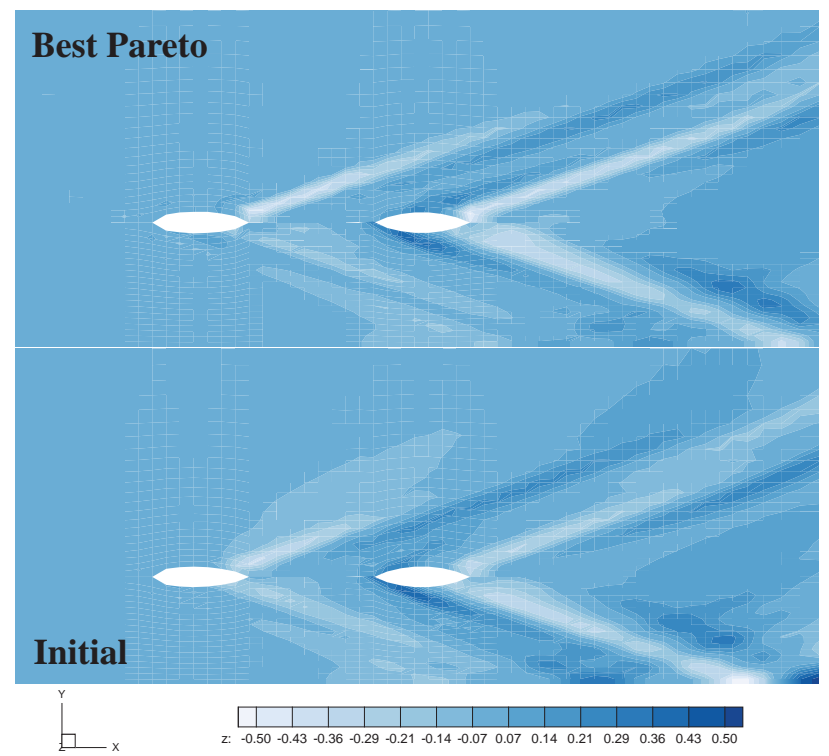

Figure 28: Wave patter comparison at Fn = 0.6. Initial Design (below) vs Best Pareto Design (above) 


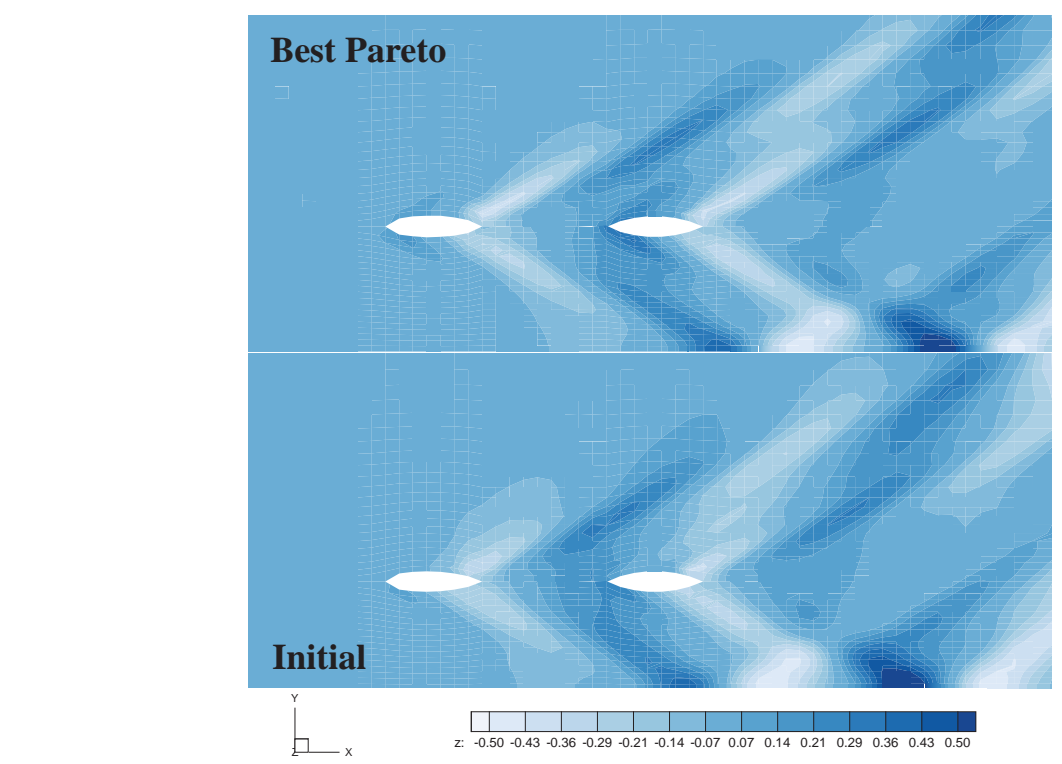

Figure 29: Wave patter comparison at Fn $=0.4$. Initial Design (below) vs Best Pareto Design (above)

\subsection{Cross comparison of the optimum hulls}

The performance of the three optimum designs (the two from the first two single-objective run and the best Pareto design from the multi-objective run) are finally compared over a wider range of Froude numbers.

The behavior of the relative resistance reduction of the three optimum hulls with respect to the reference design is shown in Figure 30. At each Froude number, the resistance over displacement ratio of the reference hull is assumed as reference value (100\%) and the relative reductions $\delta\left({ }_{T}{ }_{\Delta}\right)$ achieved by the three optimum designs are referred to it. The $1^{\text {st }}$ run optimum curve (in green), with fixed struts shapes, effectively improves resistance in the high-speed range, Fn>0.5, while its better performance vanishes in the lower speed range. Allowing for struts shape variations leads to higher reductions. However the trend of the obtained improvement is the same between the first and the second optimization cases being a direct cause of the definition of the same objective function at the same single speed. In fact, the Best Pareto design (red curve) converges to the $2^{\text {nd }}$ run optimum (blue curve) at $\mathrm{Fn}=0.6$ but reaching a better relative gain at the lower speed exploiting the multi-objective feature of the optimization algorithm.

The fractions of wave and viscous resistance in the total resistance of the reference hull are shown Figure 31 both at $\mathrm{Fn}=0.4$ and $\mathrm{Fn}=0.6$. The wave resistance of the initial hull contributes for the $49 \%$ to the total at high speed and for the $61 \%$ at lower speed respectively. The common aspect of the three optimized hulls is the marked reduction of the wave resistance fraction over the total, with the only exception of the $1^{\text {st }}$ optimization run at the lower speed. The $\mathrm{R}_{\mathrm{W}} / \Delta$ ratio of the Best Pareto Design becomes the $28 \%$ and the $23 \%$ of the total resistance of the initial hull at $\mathrm{Fn}=0.4$ and $\mathrm{Fn}=0.6$ respectively. The viscous resistance does not change considerably, since it is exclusively related to the wetted surface of the hull.

A further verification of the final result obtained by the multi-objective computation has been done by comparison with fully viscous RANSE computation. Even if the BEM over predicts the improvements with respect to that from the RANSE computations the overall trend is correctly caught; in the context of the optimization analysis framework, that is a comparative study based on relative differences among the designs, this is the main feature to be able to correctly predict in order to obtain reliable results. 


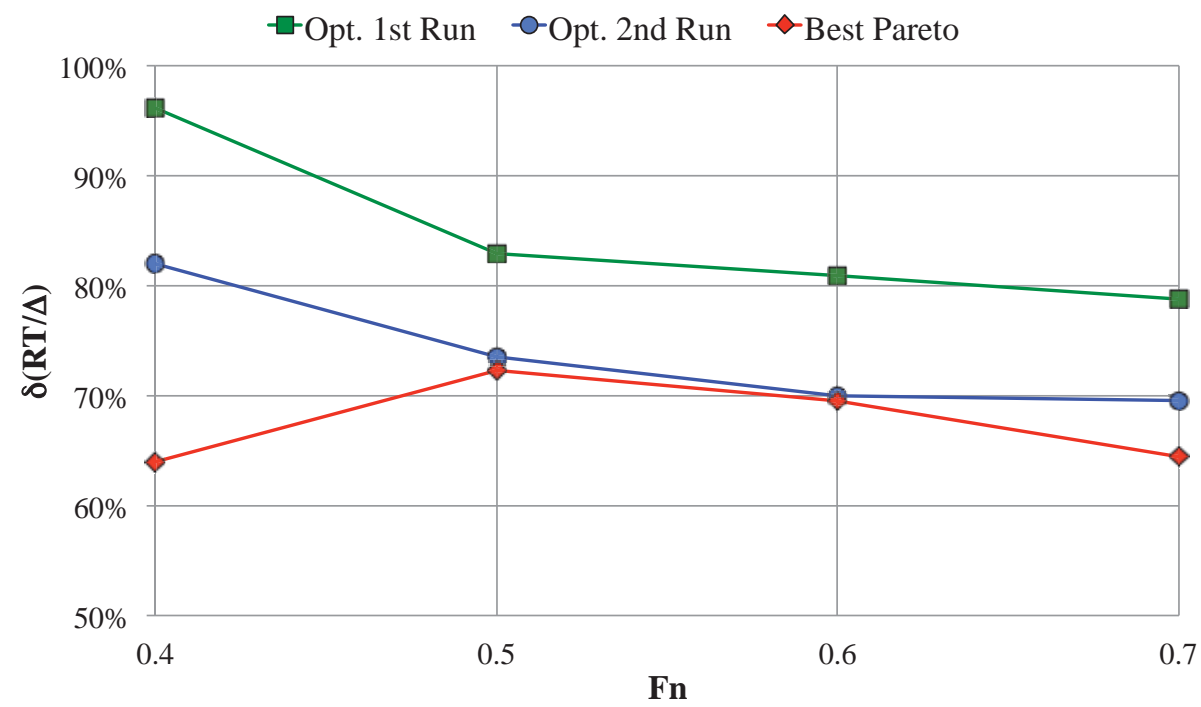

Figure 30: Percentage reductions of the total resistance over displacement $\left(\mathrm{R}_{\mathrm{T}} / \Delta\right)$ for the three optimized hulls with respect to the reference design over the entire Froude numbers range of interest

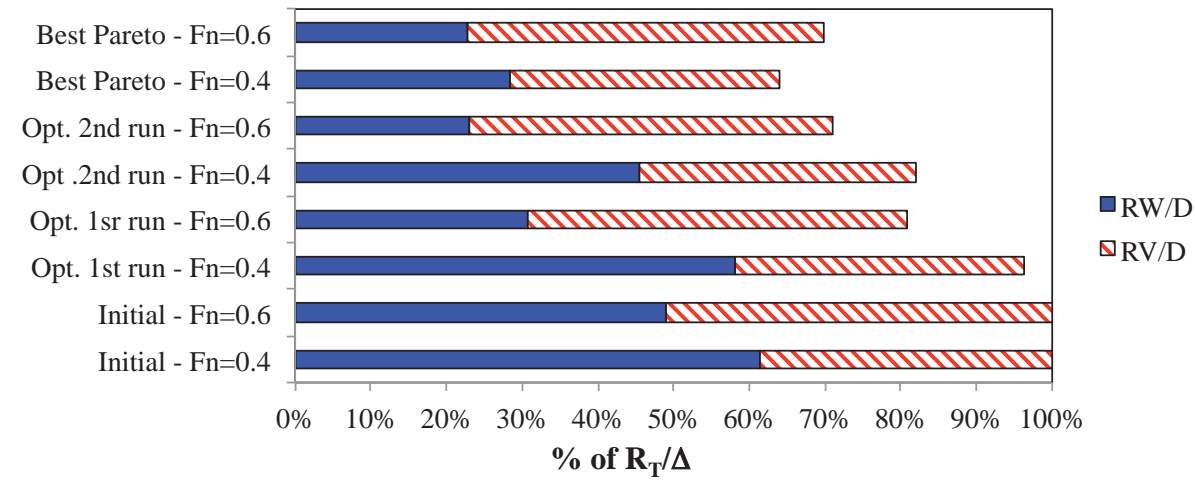

Figure 31: Fraction of wave and viscous resistance (over displacement) for the three optimum hulls with respect to the total resistance (over displacement) of the reference hull (tagged as initial in the figure).

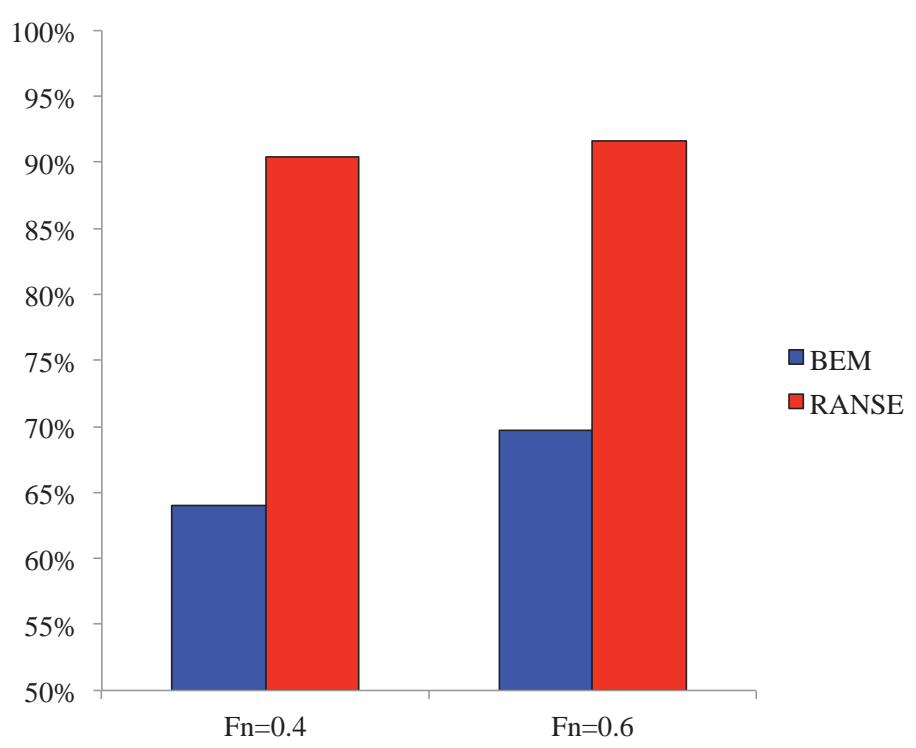

Figure 32: BEM - RANSE comparison. Percentage resistance reduction of the final optimal shape. 
In addition to the contour plot of the near-field free wave patterns, the longitudinal wave cuts at the two optimization speeds ( $\mathrm{Fn}=0.4$ and $\mathrm{Fn}=0.6)$ are shown in Figures 33 to 36. Considering that the separation of the SWATH underwater body, $s$, is the same for all the four hulls, the first longitudinal plane is located at $\mathrm{y} /(\mathrm{s} / 2)=0.0$, i.e. the symmetry plane of the complete hull; the second longitudinal plane is placed at $\mathrm{y} /(\mathrm{s} / 2)=1.5$, that is in the free surface outside the demi-hull.

Two pairs of similar wave profiles can be identified. Indeed the waves of the initial and the optimum hull obtained with the first optimization (red and green curve respectively) are almost coincident; there are only small differences in the height of the crests and hollows at the higher speed rather than in their longitudinal positions. This could be expected since the canting angle of the struts is equal between the two designs. The other pair of similar curves groups the wave profile of the optimum of the second optimization case and that of the Best Pareto design; the effect of the different strut canting angle is to shift the longitudinal position of the wave crests particularly in between the demi-hulls, where the interference effects have a stronger influence. At the relatively high Froude numbers considered for this study $(\mathrm{Fn}=0.6)$, the crests and troughs of the wave cuts are due to divergent wave trains which are very much influenced by the shape of the struts that interferes with the wave trains produced by the a rapid change of the underwater hull volume.

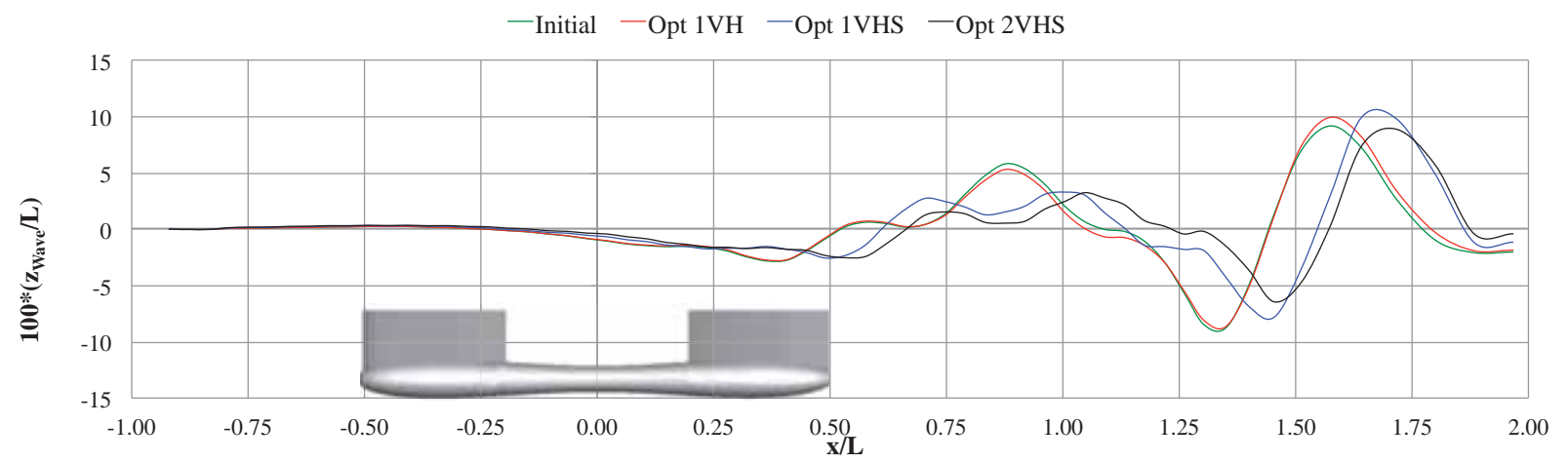

Figure 33: Longitudinal wave cuts at $\mathrm{y} /(\mathrm{s} / 2)=0, \mathrm{Fn}=0.6$. Initial hull (green), hull from Opt $1^{\text {st }}$ run (red), hull from Opt $2^{\text {nd }}$ run (blue) and Best Pareto hull (black). A generic hull is shown to clarify the location of the waves.

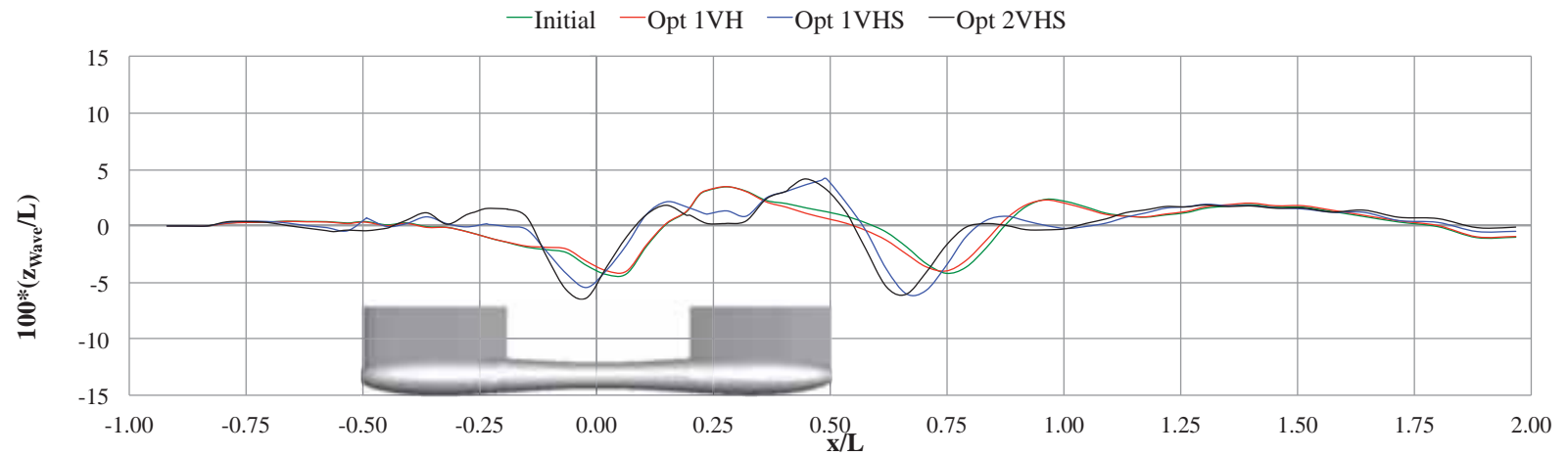

Figure 34: Longitudinal wave cuts at $\mathrm{y} /(\mathrm{s} / 2)=1.5, \mathrm{Fn}=0.6$. Initial hull (green), hull from Opt $1^{\text {st }}$ run (red), hull from Opt $2^{\text {nd }}$ run (blue) and Best Pareto hull (black). A generic hull is shown to clarify the location of the waves. 


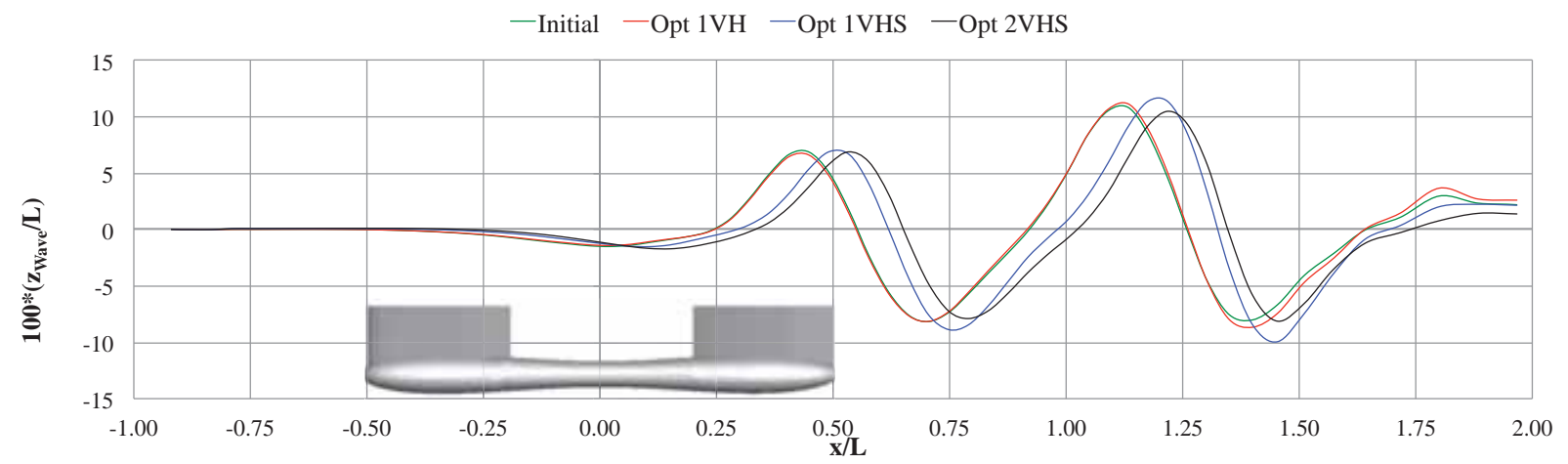

Figure 35: Longitudinal wave cuts at $\mathrm{y} /(\mathrm{s} / 2)=0, \mathrm{Fn}=0.4$. Initial hull (green), hull from Opt $1^{\text {st }}$ run (red), hull from Opt $2^{\text {nd }}$ run (blue) and Best Pareto hull (black). A generic hull is shown to clarify the location of the waves.

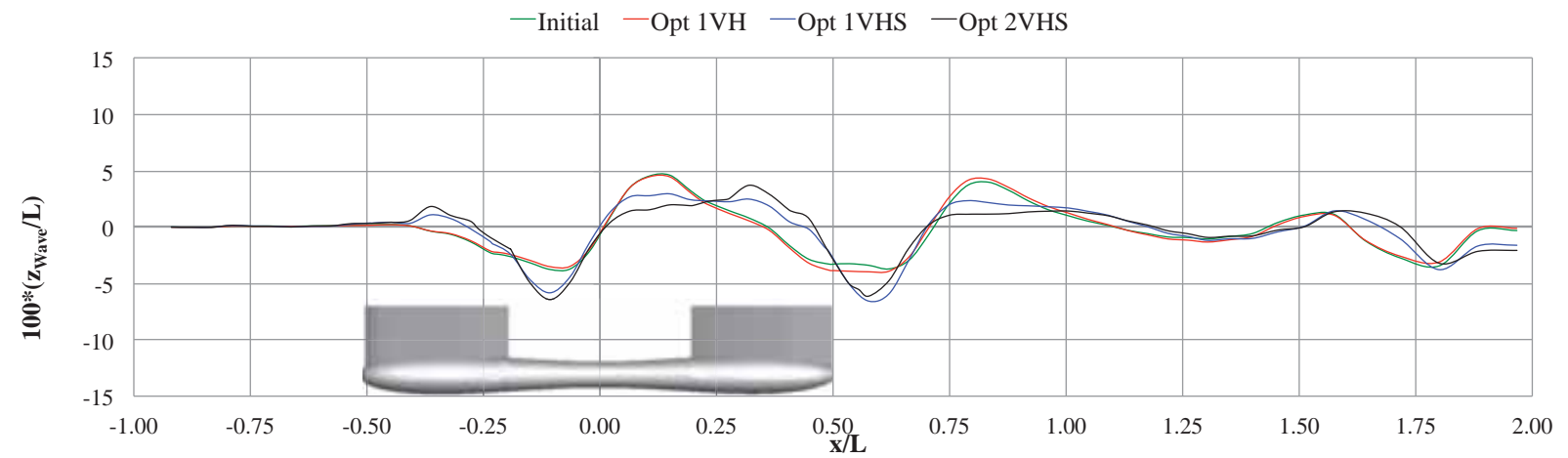

Figure 36: Longitudinal wave cuts at $\mathrm{y} /(\mathrm{s} / 2)=1.5, \mathrm{Fn}=0.4$. Initial hull (green), hull from Opt $1^{\text {st }}$ run (red), hull from Opt $2^{\text {nd }}$ run (blue) and Best Pareto hull (black). A generic hull is shown to clarify the location of the waves.

The parameters that affect the struts shape are compared in Table 4 and in Table 5 respectively for the second and for the third optimized hulls (the first optimization case does not allow any strut shape modification). For each parameter the relative difference with respect to the initial (reference) hull is reported. The canting angles for both the aft and the forward strut, $\vartheta_{A f t}$ and $\vartheta_{\text {Forw }}$, can change but of the same amount hence keeping them in-line. Negative changes of this angle correspond to an inclination of the struts towards the symmetry plane of the SWATH. This is the parameter that is able to produce the greater effect on the wave resistance reduction, as confirmed comparing results from the first and the second optimization process.

Table 4: $2^{\text {nd }}$ and $3^{\text {rd }}$ optimized hulls aft strut free parameters comparison (with respect to the initial design)

\begin{tabular}{cccccc} 
& $\vartheta_{\text {Aft }}$ & $\alpha_{\text {Entrance }}$ & $\alpha_{\text {Exit }}$ & $z_{M}$ & $x_{\text {Aft }}$ \\
\hline $2^{\text {nd }}$ & $-77.43 \%$ & $-0.01 \%$ & $-7.72 \%$ & $4.46 \%$ & $0.04 \%$ \\
$3^{\text {rd }}$ & $-98.57 \%$ & $2.74 \%$ & $-1.08 \%$ & $-2.31 \%$ & $-6.80 \%$ \\
\hline
\end{tabular}

Table 5: $2^{\text {nd }}$ and $3^{\text {rd }}$ optimized hulls forward strut free parameters comparison (with respect to the initial design)

\begin{tabular}{cccccc}
\hline & $\vartheta_{\text {Forw }}$ & $\alpha_{\text {Entrance }}$ & $\alpha_{\text {Exit }}$ & $Z_{M}$ & $x_{\text {Forw }}$ \\
\hline $2^{\text {nd }}$ & $-77.43 \%$ & $0.01 \%$ & $0.00 \%$ & $3.52 \%$ & $0.00 \%$ \\
$3^{\text {rd }}$ & $-98.57 \%$ & $0.00 \%$ & $-12.69 \%$ & $3.97 \%$ & $-13.17 \%$ \\
\hline
\end{tabular}

\section{CONCLUSIONS}

Optimal shapes of unconventional SWATH hull forms have been found and discussed in the paper, with special regards to twin struts shape and canting angle and to the effect of a multiple velocity profile on the optimum shape. The hull shapes were found by a computer driven procedure based on fully parametric geometry definition, a low order potential flow solver with linearized free surface condition and a multi-objective 
differential evolution optimization algorithm. Total resistance has been calculated adding the potential flow pressure resistance to the viscous resistance estimated for the given hull wetted surface assuming a constant form factor. Three different hull form optimization studies have been considered. Two single objective optimization runs have been performed at $F n=0.6$ : in the first, the shape of the underwater hull only has been allowed to change including the ellipticity factor of the transverse sections with respect to previously published results; the second optimization study adds the effect of the variable shape and canting angle of the twin struts. These studies are preliminary to the final multi-objective hydrodynamic shape optimization computation. This last optimization considered the total resistance of the SWATH at two very different speeds Fn=0.6 and Fn=0.4, where a distinct hump was noted from the analysis of the wave resistance calculated for high speed optimum hull forms.

The following conclusions are derived from this numerical study: (1) the struts geometry can contribute to reduce the total resistance from $19 \%$, achievable considering only the shape of the underwater hull, up to $29 \%$, contributing for about two third of the total; (2) including strut shape variations lead to a design able to maintain the relative resistance reduction also at lower speeds; (3) the hull shape resulting from the multi-objective optimization is able to optimize the resistance reduction over the whole range of speeds, i.e. Fn=0.4 0.7; (4) the struts canting angle has a major effect on the interference of the waves generated by the forward and the aft struts being an essential parameter in the design of efficient SWATH vessels.

\section{ACKNOWLEDGEMENTS}

This was has been possible due to the support of ONR through Grant N000141310834 to MIT-iShip lab, P.I. Stefano Brizzolara.

\section{REFERENCES}

Biliotti, I., Brizzolara, S., Viviani, M., Vernengo, G., Ruscelli, D., Galliussi, M., Guadalupi, D. \& Manfredini, A. (2011, September). Automatic parametric hull form optimization of fast naval vessels. In $11^{\text {th }}$ international conference on fast sea transportation (FAST), Honolulu, Hawaii, USA.

Bonfiglio, L, Brizzolara, S (2013). "Influence of Viscosity on Radiation Forces: a Comparison between Monohull, Catamaran and SWATH”, ISOPE 2013, Proc $23^{\text {rd }} 17^{\text {th }}$ Int Offshore and Polar Eng Conf, Anchorage, Alaska, USA, ISOPE, 3, 718-725. (ISBN 978-1880653 99-9).

Brizzolara, S (2004). "Parametric Optimization of SWAT-Hull Forms by a Viscous-Inviscid Free Surface Method Driven by a Differential Evolution Algorithm". 25 $5^{\text {th }}$ Symposium on Naval Hydrodynamics, Saint John's (CA), National Academies Press, 5, 1-18.

Brizzolara, S, Bruzzone, D, Cassella, P, Scamardella, I, and Zotti, I (1998). "Wave Resistance and Wave Pattern for High Speed Crafts; Validation of Numerical Results by Model Tests," Proc $22^{\text {nd }}$ Symp Naval Hydrodyn, Washington, DC, USA, 69-83.

Brizzolara S., Bruzzone D (2007). Hydrodynamic Assessment and Optimization of New Fast Foil Assisted SWAMH. In Proceedings of the $11^{\text {th }}$ International Symposium on Practical Design of Ships and Other Floating Strutures, PRADS 2007. Houston, Oct 2007, vol. 1, p. 205-211, ISBN/ISSN: 0-943870-04-6

Brizzolara, S, Curtin, T, Bovio, M, Vernengo, G (2011). "Concept design and hydrodynamic optimization of an innovative SWATH USV by CFD methods”, Ocean Dynamics, Elsevier, 62(2), 227-237. (DOI:10.1007/s10236- 
011-0471-y).

Brizzolara, S, and Vernengo, G (2011). "Automatic computer driven optimization of innovative hull forms for marine vehicles". 10th WSEAS International Conference on Applied Computer and Applied Computational Science, ACACOS'11, pp. 273-278, 2011.

Brizzolara S., Chryssostomidis C. (2014). Numerical Predictions of the relative motion of a SWATH ASV and an AUV in waves. A true step forward towards accurate L\&R simulations. In Proceedings of ASNE International Launch \& Recovery Symposium, November 19-20, 2014, Linthicum (MD)

Brizzolara, S., Vernengo, G., Bonfiglio, L., Bruzzone, D. (2015) Comparative performance of optimum high speed SWATH and Semi-SWATH in calm water and in waves. Transactions - Society of Naval Architects and Marine Engineers, 123, pp. 273-286.

Bruzzone D (1994). Numerical evaluation of the steady free surface waves. CFD Workshop, Tokyo, 1994, Ship Res. Inst. Tokyo, Vol I, pp. 126-134.

Deb, K, Agrawal, S, Pratap, A, Meyarivan, T (2000). "A fast elitist non- dominated sorting genetic algorithm for multi-objective optimi- zation: NSGA-II", in Schoenauer, M, et al (eds), "Parallel problem solving from nature", Springer, Paris, pp 849-858.

Esteco (2014). modeFRONTIER, Version 4.4.4, Trieste, Italy, http://www.esteco.it.

Harries, S (1998). "Parametric Design and Hydrodynamic Optimiza- tion of Ship Hull Forms", PhD Thesis, Technische Universität Berlin, Berlin, Germany.

Lang T.G. (1986). SSP Kaimalino; Conception, Developmental History, Hurdles and Success. Presented at the ASME Winter Annual Meeting, Anaheim, California - December 7-12.

Molland A F, Turnock, S R, and Hudson, D A (2011). "Ship Resistance and Propulsion: Practical Estimation of Ship Propulsive Power", Cambridge University Press, 568 pp.

Navatek (2015) SWATH I and II, from website: www.navatekltd.com

NOAA (2015). Ship Ferdinand R. Hassler http://www.moc.noaa.gov/fh/

Papanikolaou, A, Zaraphonitis, G, and Androulakakis, M (1991). "Preliminary Design of a High-Speed SWATH Passenger/Car Ferry," Mar Technol, 28(3), 129-141.

Sobol, I M (1967). "Distribution of Points in a Cube and Approxi-mate Evaluation of Integrals," USSR Comput Math Math Phys, 7, 86-112. (DOI:10.1016/0041-5553(67)90144-9).

Scragg CA, Reed AM, Wyatt DC, Ratcliffe TJ (1998). Hull form development of the sea shadow and applications of the technology of monohulls. SNAME Transactions, Vol. 106, pp. $443-483$.

Pelly D. (2009). Silver Cloud. Boat International, April 2009

Vernengo, G, Brizzolara, S, Bruzzone, D (2015). "Resistance and Seakeeping Optimization of a Fast Multihull Passenger Ferry", Int J Offshore and Polar Eng, IJOPE, 25(1), 1-9. (ISSN 1053-5381). 


\section{List of Figures}

Figure 1: Parametric definition of the B-Spline curve used to generate the shape of the underwater hull of the SWATH. Allowed horizontal and vertical motions of the points are highlighted green and red arrows respectively.

Figure 2: Strut parametric section definition. Allowed movements of points are highlighted as well as possible angle changes.

Figure 3: Reference system for the strut rotation by the canting angle, $\vartheta$

Figure 4: Initial hull shape. Ellipticity factor $\mathrm{f}=1.4$

Figure 5: Ellipticity factor variation $(\mathrm{f}=1.2)$

Figure 6: Ellipticity factor variation $(\mathrm{f}=1.6)$

Figure 7: Ellipticity factor variation $(\mathrm{f}=1.8)$

Figure 8: Upright struts (null canted angle)

Figure 9: Increased struts canted angle $\left(\vartheta=10^{\circ}\right)$

Figure 10: Increased struts canted angle $\left(\vartheta=20^{\circ}\right)$

Figure 11: Increased struts canted angle $\left(\vartheta=30^{\circ}\right)$

Figure 12: Forward strut entrance angle $\left(\alpha=30^{\circ}\right)$

Figure 13: Forward strut entrance angle $\left(\alpha=70^{\circ}\right)$

Figure 14: Numeric-Experimental comparison of the total resistance coefficient for a single -strut SWATH

Figure 15: Example of free surface panel mesh sensitivity. Resistance relative variation is monitored with respect to changes in the number of waterline element and lateral dimension of the first element close to the hull.

Figure 16: Extract of the final hull and free surface panel mesh configuration

Figure 17: Optimization history. Single-Objective run. Fixed struts shapes

Figure 18: Optimization history. Single-Objective run. Struts shapes free to change

Figure 19: Perspective/front view of the initial hull shape (demi-hull)

Figure 20: Perspective/front view of the optimized hull from the 1st run: Fixed struts optimization. Only one demi-hull is presented.

Figure 21: Perspective/front view of the optimized hull from the 2nd run: Free-to-change struts optimization.

Figure 22: Wave pattern of the initial hull $(\mathrm{Fn}=0.6)$

Figure 23: Wave pattern of the optimized design of the $1^{\text {st }}$ run $(\mathrm{Fn}=0.6)$

Figure 24: Wave pattern of the optimized design of the 2nd run $(\mathrm{Fn}=0.6)$

Figure 25: Multi-objective function scatter diagram with all feasible designs. Highlighted: Initial design (green square). Best Pareto design (red rhombus). Best design for $\mathrm{Fn}=0.6$ (blue triangle). Best design for $\mathrm{Fn}=0.4$ (pink triangle).

Figure 26: Zoom of the multi-objective function scatter diagram with all feasible designs close to the Pareto

Frontier. Highlighted: Initial design (green square). Best Pareto design (red rhombus). Best design for $\mathrm{Fn}=0.6$ (blue triangle). Best design for $\mathrm{Fn}=0.4$ (pink triangle).

Figure 27: Perspective view of the Best Pareto hull from the multi-objective run (demi-hull).

Figure 28: Wave patter comparison at $\mathrm{Fn}=0.6$. Initial Design (above) vs Best Pareto Design (below)

Figure 29: Wave patter comparison at Fn =0.4. Initial Design (above) vs Best Pareto Design (below)

Figure 30: Percentage reductions of the total resistance over displacement (RT/D) for the three optimized hulls with respect to the reference design over the entire Froude numbers range of interest

Figure 31: Fraction of wave and viscous resistance (over displacement) for the three optimum hulls with respect to the total resistance (over displacement) of the reference hull (tagged as initial in the figure).

Figure 32: BEM - RANSE comparison. Percentage resistance reduction of the final optimal shape.

Figure 33: Longitudinal wave cuts at $\mathrm{y} /(\mathrm{s} / 2)=0, \mathrm{Fn}=0.6$. Initial hull (red), hull from Opt 1st run (green), hull 
from Opt 2nd run (black) and Best Pareto hull (blue). A generic hull is shown to clarify the location of the waves.

Figure 34: Longitudinal wave cuts at $y /(\mathrm{s} / 2)=1.5, \mathrm{Fn}=0.6$. Initial hull (red), hull from Opt 1st run (green), hull from Opt 2nd run (black) and Best Pareto hull (blue). A generic hull is shown to clarify the location of the waves.

Figure 35: Longitudinal wave cuts at $\mathrm{y} /(\mathrm{s} / 2)=0, \mathrm{Fn}=0.4$. Initial hull (red), hull from Opt 1st run (green), hull from Opt 2nd run (black) and Best Pareto hull (blue). A generic hull is shown to clarify the location of the waves.

Figure 36: Longitudinal wave cuts at $\mathrm{y} /(\mathrm{s} / 2)=1.5, \mathrm{Fn}=0.4$. Initial hull (red), hull from Opt 1st run (green), hull from Opt 2nd run (black) and Best Pareto hull (blue). A generic hull is shown to clarify the location of the waves.

\section{List of Tables}

Table 1: Technical data of the unconventional SWATH.

Table 2: List of parameters used to generate the SWATH model.

Table 3: List of the optimization constraints with reference values and relative tolerances.

Table 4: 2nd and 3rd optimized hulls aft strut free parameters comparison (with respect to the initial design).

Table 5: 2nd and 3rd optimized hulls forward strut free parameters comparison (with respect to the initial design). 